\title{
Excursion decompositions for SLE and Watts' crossing formula
}

\author{
Julien Dubédat*
}

27th October 2018

\begin{abstract}
It is known that Schramm-Loewner Evolutions (SLEs) have a.s. frontier points if $\kappa>4$ and a.s. cutpoints if $4<\kappa<8$. If $\kappa>4$, an appropriate version of $\operatorname{SLE}(\kappa)$ has a renewal property: it starts afresh after visiting its frontier. Thus one can give an excursion decomposition for this particular $\operatorname{SLE}(\kappa)$ "away from its frontier". For $4<\kappa<8$, there is a two-sided analogue of this situation: a particular version of $\operatorname{SLE}(\kappa)$ has a renewal property w.r.t its cutpoints; one studies excursion decompositions of this SLE "away from its cutpoints". For $\kappa=6$, this overlaps Virág's results on "Brownian beads". As a by-product of this construction, one proves Watts' formula, which describes the probability of a double crossing in a rectangle for critical plane percolation.
\end{abstract}

Schramm-Loewner Evolutions (SLEs) are a family of growth processes in simply connected plane domains. Their conformal invariance properties make them natural candidates to describe the scaling limit of critical plane systems, that are generally conjectured to converge to a conformally invariant limit. This convergence has been rigorously established in several cases: for instance, the scaling limit of the Loop-Erased Random Walk (resp. the Peano curve of the Uniform Spanning Tree) is $\operatorname{SLE}(2)$ (resp. SLE(8)) (see [12]), and the scaling limit of critical percolation interfaces is $\operatorname{SLE}(6)$ (see [20]).

The qualitative features of SLE depend crucially on the value of the $\kappa$ parameter. The growth process is generated by a continuous path, the trace (see 18). This path is a.s. simple if $\kappa \leq 4$; if $\kappa>4$, it is no longer the case, and SLE has a non-trivial frontier ([18]). Furthermore, SLE has cutpoints if $4<\kappa<8$ (see [1).

In 22], Virág shows that the Brownian Excursion (Brownian motion in the upper half-plane, started from 0 and conditioned not to hit the real line again) can be decomposed in "beads", i.e. portions of the Brownian excursion between

*Université Paris-Sud 
two successive cutpoints. This decomposition can be phrased in terms similar to Itô's theory of Brownian excursions.

For SLE, one also has a Markov property and conformal invariance, so it is quite natural to look for similar decompositions w.r.t. loci with an intrinsic geometrical definition. We will see that such decompositions exist (for suitably conditioned SLEs) for frontier points and cutpoints.

While considering restriction formulas in [7, in relation with duality conjectures, it appeared that a particular version of $\operatorname{SLE}(\kappa)$, namely $\operatorname{SLE}(\kappa, \kappa-4)$, played a special role. For $\kappa>4, \operatorname{SLE}(\kappa)$ a.s. swallows any real point; and $\operatorname{SLE}_{\left(0,0^{+}\right)}(\kappa, \kappa-4)$ can be viewed as an $\operatorname{SLE}(\kappa)$ "conditioned" not to hit the positive half-line. As this event has zero probability, a little care (and precision) is required. This process, run until infinity, has a right-boundary that is a simple path connecting 0 and $\infty$ in $\mathbb{H}$; this right-boundary is conjectured to be identical in law to an $\operatorname{SLE}\left(\kappa^{\prime}, \kappa^{\prime} / 2-2\right)$, where $\kappa \kappa^{\prime}=16$.

Looking at the right-boundary as a path, it is quite natural to consider the conditional law of $\operatorname{SLE}(\kappa, \kappa-4)$ given an initial portion of its right-boundary. A point on this (final) right-boundary will never be swallowed again; so the conditional SLE $(\kappa, \kappa-4)$ avoids the already completed right-boundary, and also avoids the positive half-line. It turns out that, after taking the image under a conformal equivalence, the completed right-boundary and the positive half-line play exactly the same role. This suggests that the future of $\operatorname{SLE}(\kappa, \kappa-4)$ after a frontier time (i.e. a time at which the trace is on the final right-boundary) is again $\operatorname{SLE}(\kappa, \kappa-4)$ in the remaining domain, a renewal property similar to the Markov property of SLE. We will prove that one can define a "local time" for time spent by $\operatorname{SLE}(\kappa, \kappa-4)$ on its right-boundary; subordinating the $\operatorname{SLE}(\kappa, \kappa-4)$ by the inverse of this local time (which is a stable subordinator with index $(1 / 2+2 / \kappa))$, one gets a hull-valued Lévy process. We will also give an excursion decomposition with respect to the right-boundary (i.e. we will describe the law of $\operatorname{SLE}(\kappa, \kappa-4)$ between two successive frontier times). As a by-product of this construction, a one-parameter extension of Pitman's $(2 M-X)$ theorem is derived.

As frontier points, cutpoints form a locus with an intrinsic geometrical definition. As pointed out by Virág, the Brownian excursion (i.e. planar Brownian motion started from 0 and conditioned not to visit the lower half-plane) can be decomposed into "beads" (portions of the Brownian excursion between successive cutpoints). This decomposition relies on the conformal invariance of Brownian motion, and the Strong Markov property.

When $4<\kappa<8$, SLE is known to have cutpoints (the corresponding cuttimes have a.s. Hausdorff dimension $(2-\kappa / 4)$, see [1]). By analogy with the onesided construction, where $\operatorname{SLE}(\kappa, \kappa-4)$ turned out to be well suited to the study of the right-boundary, the structure of cut-times for $\mathrm{SLE}_{\left(0,0^{-}, 0^{+}\right)}(\kappa, \kappa-4, \kappa-4)$ is particularly nice. This is due to the fact that $\operatorname{SLE}_{\left(0,0^{-}, 0^{+}\right)}(\kappa, \kappa-4, \kappa-4)$ can be viewed as $\operatorname{SLE}(\kappa)$ "conditioned not to hit $\mathbb{R}^{*}$ ", via an appropriate conditioning 
procedure. The construction of the local time of cutpoints is more involved than that of "frontier local time" (based on local times for Bessel processes), but it can be carried out explicitly. Subordinating the SLE $(\kappa, \kappa-4, \kappa-4)$ process by the inverse of this local time leads also to a hull-valued Lévy process; an Itô-type result for the "bead process" is also derived. In the particular case $\kappa=6$, as the Brownian excursion and $\operatorname{SLE}(6,2,2)$ can be seen as realizations of the (unique) restriction measure with exponent 1 , these results partially overlap those in [22].

In the first section, we derive some properties of $\operatorname{SLE}(\kappa, \kappa-4)$ and $\operatorname{SLE}(\kappa, \kappa-$ $4, \kappa-4)$ processes that will be used later, using mainly stochastic calculus. The second section is devoted to the study of $\operatorname{SLE}(\kappa, \kappa-4)$ in relation with its right-boundary. We define a Markov process measuring the distance to the boundary; the local time at 0 of this process defines a "frontier local time". The third section develops analogous results in the two-sided case (i.e. in relation with cutpoints), beginning with a systematic reinterpretation of the one-sided situation in terms of Doob $h$-transforms/Girsanov densities. Though the line of reasoning is essentially parallel, details are much more intricate in the two-sided case. In the last section, we use some of the previous results on $\operatorname{SLE}(6,2,2)$ to prove Watts' formula, which describes the probability of the existence of a double crossing in a rectangle, in the scaling limit of critical percolation.

Acknowledgments. I wish to thank Wendelin Werner for his help and advice along the preparation of this paper, as well as Marc Yor for stimulating conversations.

\section{Introduction and notations}

In this section, we briefly recall the definition and elementary properties of SLE processes, and collect first properties of SLE $(\kappa, \kappa-4)($ resp. SLE $(\kappa, \kappa-4, \kappa-4))$ processes.

\subsection{Chordal SLE and $\operatorname{SLE}(\kappa, \underline{\rho})$ processes}

For general background on SLE processes, introduced by Oded Schramm in 19, see [18, 24. In this article, we will consider only chordal SLEs, i.e. SLEs that grow from one boundary point to another boundary point in a simply connected plane domain.

Chordal Loewner equations are a device that encode a growth process in a plane simply connected domain by a real-valued process. More precisely, consider the upper half-plane $\mathbb{H}=\{z: \Im z>0\}$ (without loss of generality since, by Riemann's mapping theorem, any simply connected domain other than $\mathbb{C}$ is 
conformally equivalent to $\mathbb{H}$ ), a function $w: \mathbb{R}^{+} \mapsto \mathbb{R}$, and the family of ODEs:

$$
\partial_{t} g_{t}(z)=\frac{2}{g_{t}(z)-w_{t}}
$$

with initial condition $g_{0}(z)=z, z \in \mathbb{H}$. The solution of any of these ODEs is defined up to explosion time $\tau_{z}$ (possibly infinite). Then, for $t \geq 0$, let

$$
K_{t}=\overline{\left\{z \in \mathbb{H}: \tau_{z}<t\right\}} .
$$

The increasing family $\left(K_{t}\right)$ of compact subsets of $\overline{\mathbb{H}}$ is such that

$$
g_{t}(z)=z+\frac{2 t}{z}+O\left(z^{-2}\right)
$$

is the unique conformal equivalence $\mathbb{H} \backslash K_{t} \rightarrow \mathbb{H}$ with asymptotic expansion at infinity $g_{t}(z)=z+o(1)$ (hydrodynamic normalization). The coefficient $2 t$ in this expansion is by definition the half-plane capacity $\operatorname{cap}\left(K_{t}\right)$ of $K_{t}$. This construction sets up a bijection between real-valued continuous processes and increasing families of compact sets $\left(K_{t}\right)$ such that $\operatorname{cap}\left(K_{t}\right)=2 t$, under a "local growth" condition (see e.g. 24]).

If $W / \sqrt{\kappa}$ is a standard (real) Brownian motion, the associated (random) families of hulls $\left(K_{t}\right)$ and conformal equivalences $\left(g_{t}\right)$ define the chordal SchrammLoewner Evolution in $(\mathbb{H}, 0, \infty)$ with parameter $\kappa$, in short $\operatorname{SLE}(\kappa)$. Note that, as a consequence of Brownian scaling, $\left(\lambda^{-1} K_{\lambda^{2} t}\right)_{t \geq 0}$ as the same law as $\left(K_{t}\right)$ for any positive $\lambda$. Since the dilatations $z \mapsto \lambda z, \lambda>0$, are the only conformal automorphisms of $(\mathbb{H}, 0, \infty)$, one can define $\operatorname{SLE}(\kappa)$ in any simply connected domain $(D, a, b)$, where $a$ and $b$ are two distinct boundary points, as the image of chordal $\operatorname{SLE}(\kappa)$ in $(\mathbb{H}, 0, \infty)$ as defined above under any conformal equivalence between $(\mathbb{H}, 0, \infty)$ and $(D, a, b)$.

If $\left(K_{t}\right)$ is a Loewner chain associated with an $\operatorname{SLE}(\kappa)$ process, then for any $s \geq 0,\left(g_{s}\left(K_{t+s} \backslash K_{s}\right)-W_{s}\right)_{t}$ defines a chordal $\operatorname{SLE}(\kappa)$ process in $(\mathbb{H}, 0, \infty)$ independent from $\left(K_{t}\right)_{t \leq s}$. One can see this as an independent increment property, where the "increment" is the conformal equivalence $\left(g_{s}-W_{s}\right)$.

For any $\kappa>0$, there exists a continuous process $\gamma$ taking values in $\overline{\mathbb{H}}$ that generates the hulls $\left(K_{t}\right)$ of $\operatorname{SLE}(\kappa)$ in the following sense: a.s., $\mathbb{H} \backslash K_{t}$ is the unbounded connected component of $\mathbb{H} \backslash \gamma_{[0, t]}$ for any $t \geq 0$; this process $\gamma$ is the trace of the SLE (see [18, 12] for the case $\kappa=8$ ).

If $\kappa \leq 4$, the trace is a.s. simple; this is no longer the case if $\kappa>4$ (see [18]). Hence, if $\kappa>4$, the outer boundary of an $\operatorname{SLE}(\kappa)$ hull $K_{t}$ is strictly included in the image of the trace $\gamma_{[0, t]}$. Furthermore, if $4<\kappa<8$, an $\operatorname{SLE}(\kappa)$ hull $K_{t}$ has cutpoints with positive probability ([1]).

Other probability measures on processes can be translated in laws on increasing families of hulls by means of Loewner equations. For $n \in \mathbb{N}$ and a 
family of parameters $\underline{\rho}=\left(\rho_{1}, \ldots, \rho_{n}\right)$, consider the SDEs

$$
\left\{\begin{aligned}
d W_{t} & =\sqrt{\kappa} d B_{t}+\sum_{i=1} \frac{\rho_{i} d t}{W_{t}-Z_{t}^{(i)}} \\
d Z_{t}^{(i)} & =\frac{2 d t}{Z_{t}^{(i)}-W_{t}}
\end{aligned}\right.
$$

for $i=1 \ldots n$, where $B$ is a standard Brownian motion, with initial conditions $W_{0}=0, Z_{0}^{(i)}=x_{i} \in \mathbb{R}$. Then the image of the process $W$ under the Loewner equations define $\operatorname{SLE}(\kappa, \underline{\rho})$. The $\operatorname{SLE}(\kappa, \rho)$ processes were introduced in [1]; see also [25, 7]. In fact, we will use only the cases $n=1,2$; questions of definiteness will be discussed when needed.

\subsection{Properties of $\operatorname{SLE}(\kappa, \kappa-4)$ and $\operatorname{SLE}(\kappa, \kappa-4, \kappa-4)$ pro- cesses}

In this section, we collect some of the properties of $\operatorname{SLE}(\kappa, \kappa-4)$ and $\operatorname{SLE}(\kappa, \kappa-$ $4, \kappa-4)$ that we will use later. Let us stress that almost all of these properties seem to have no direct equivalent for other choices of the $\rho$ parameter. Let $\kappa>4$ be fixed, and $d=1-\frac{4}{\kappa}$. Recall that a $\operatorname{Beta}(a, b)$ law is a probability law supported on $[0,1]$ with density:

$$
B(a, b)^{-1} \mathbf{1}_{x \in(0,1)} x^{a-1}(1-x)^{b-1}
$$

where $B(a, b)=\Gamma(a) \Gamma(b) / \Gamma(a+b)$, and $a, b$ are positive.

Proposition 1. Let $\left(W_{t}, O_{t}\right)_{t}$ be the driving process of an $\operatorname{SLE}(\kappa, \kappa-4)$ starting from $(0, y), y>0$ and $\left(\mathcal{F}_{t}\right)$ the associated natural filtration. For $x \in(0, y)$, let $\tau_{x} \in(0, \infty]$ be the swallowing time of $x$. Then:

(i) $\mathbb{P}\left(\tau_{x}=\infty\right)=(x / y)^{d}$.

(ii) The law of the SLE conditionally on $\left\{\tau_{x}=\infty\right\}$ is that of an $\operatorname{SLE}(\kappa, \kappa-4)$ starting from $(0, x)$.

(iii) Let $Z$ be the rightmost swallowed point of $(0, y)$. Then $Z / y$ is an $\mathcal{F}_{\infty}$ measurable sample from the $\operatorname{Beta}(d, 1)$ law.

(iv) Let $\tau_{Z}$ be the a.s. finite $\mathcal{F}_{\infty}$-measurable random time at which $Z$ is swallowed. For $z \in(0,1)$, let $Q(z,$.$) denote the probability measure on bivariate$ $\left(W_{t}, O_{t}\right)$ processes obtained as the concatenation of an $\operatorname{SLE}(\kappa,-4)$ starting from $(0, z)$, which is defined up to time $\tau_{z}$, and an independent $\operatorname{SLE}(\kappa, \kappa-4)$ starting from $\left(W_{\tau_{z}}, W_{\tau_{z}}^{+}\right)$. Then $\omega \mapsto Q(Z(\omega)$,. $)$ defines a regular conditional probability of the original $\mathrm{SLE}_{(0, y)}(\kappa, \kappa-4)$ process w.r.t. $\sigma(Z)$.

Proof. (i) It is immediate to check that the semimartingale:

$$
t \mapsto\left(\frac{g_{t}(x)-W_{t}}{O_{t}-W_{t}}\right)^{d},
$$

which is defined up to $\tau_{x}$, is a local martingale taking values in $[0,1]$, hence is a martingale. If $\tau_{x}<\infty$, as $y$ is never swallowed, it appears that as $t \nearrow \tau_{x}$, this 
martingale goes to zero (as the extremal distance between $g_{t}^{-1}\left(\left(W_{t}, g_{t}(x)\right)\right)$ and $(y, \infty)$ in $\mathbb{H} \backslash K_{t}$ goes to infinity). Conversely, if $\tau_{x}=\infty$, this extremal distance goes to 0 (by transience of the SLE), so the limit of the martingale is 1 . One concludes with the optional stopping theorem (as e.g. in [24],3.2).

(ii) See 7], Section 5 .

(iii) The density follows from (i), since, from the definition of $Z$, one has : $\{Z<x\}=\left\{\tau_{x}=\infty\right\}$.

(iv) We are interested in the law of the SLE conditioned on Z. Suppose $Z \in(z-\varepsilon, z)$ for some $z \in(0,1]$; then $z$ is not swallowed, so from (ii), an $\operatorname{SLE}_{(0, y)}(\kappa, \kappa-4)$ conditionally on $Z \in(z-\varepsilon, z)$ has the law of an $\operatorname{SLE}_{(0, z)}(\kappa, \kappa-$ 4) conditionally on $Z>z-\varepsilon$. We argue as in [7], Section 5; if $\mathbf{P}$ denotes the original probability measure (corresponding to a $\operatorname{SLE}(\kappa, \kappa-4)$ starting from $(0, y))$ and $\mathbf{Q}=\mathbf{P}(. \mid Z \in(z-\varepsilon, z))$, then there exists a process $\tilde{B}$, which is a standard Brownian motion under $\mathbf{Q}$, such that:

$d W_{t}=\sqrt{\kappa} d \tilde{B}_{t}+\frac{\kappa-4}{g_{t}(z)-W_{t}} d t-(\kappa-4) \frac{g_{t}(z)-g_{t}(z-\varepsilon)}{\left(g_{t}(z)-W_{t}\right)^{2}} U_{t}^{-4 / \kappa}\left(1-U_{t}^{1-4 / \kappa}\right)^{-1} d t$

where $U_{t}=\left(g_{t}(z-\varepsilon)-W_{t}\right) /\left(g_{t}(z)-W_{t}\right)$. As $\varepsilon \searrow 0$, the drift term in this equation tends to $-4 /\left(g_{t}(z)-W_{t}\right) d t$. This limit $\operatorname{SDE}$ defines an $\operatorname{SLE}_{(0, z)}(\kappa,-4)$ process; this indicates that an $\operatorname{SLE}_{(0, y)}(\kappa, \kappa-4)$ conditioned by $Z \in d z$, and stopped on swallowing $Z$ at time $\tau_{Z}$, is an $\operatorname{SLE}_{(0, z)}(\kappa,-4)$ stopped at time $\tau_{z}$. Note that this last process is well defined up to time $\tau_{z}$, and that in this case $\left(g_{t}(z)-W_{t}\right) / \sqrt{\kappa}$ is a standard $\operatorname{BES}(d)$ process stopped on hitting 0.

We make the previous limiting argument a bit more precise. Let $\varepsilon_{0}>0$ be fixed. Consider a process $R=\left(R_{1}, R_{2}, R_{3}\right)$ taking values in the Banach space $C_{0}\left(\left[0, \varepsilon_{0}\right], \mathbb{R}^{3}\right)$ (for uniform convergence w.r.t. some norm of $\mathbb{R}^{3}$ ), that satisfies the following SDE:

$$
d R_{t}=(\sqrt{\kappa}, 0,0) d \tilde{B}_{t}+\left(b_{1}\left(R_{t}\right), \frac{2}{R_{2, t}-R_{1, t}}, \frac{2}{R_{3, t}-R_{1, t}}\right) d t
$$

with initial conditions $R_{0}(\varepsilon)=(0, z-\varepsilon, z)$. The drift term $b_{1}(r)$ is defined by:

$$
b_{1}(r)=\frac{\kappa-4}{r_{3}-r_{1}}-(\kappa-4) \frac{r_{3}-r_{2}}{\left(r_{3}-r_{1}\right)^{2}}\left(\frac{r_{2}-r_{1}}{r_{3}-r_{1}}\right)^{-4 / \kappa}\left(1-\left(\frac{r_{2}-r_{1}}{r_{3}-r_{1}}\right)^{1-4 / \kappa}\right)^{-1}
$$

with $b_{1}(r)(0)=\lim _{\varepsilon \searrow 0} b_{1}(r)(\varepsilon)=-4 /\left(r_{3}-r_{1}\right)$. Now let $\eta>0$ be an arbitrary small number, and let $\Delta_{\eta}$ be the following closed subset of $C_{0}\left(\left[0, \varepsilon_{0}\right], \mathbb{R}^{3}\right)$ :

$$
\Delta_{\eta}=\left\{\left(r_{1}, r_{2}, r_{3}\right): r_{1} \leq r_{2} \leq r_{3}, r_{3}(\varepsilon)-r_{2}(\varepsilon) \leq \varepsilon, r_{2}-r_{1} \geq \eta\right\}
$$

Let $b_{2}(r)=2 /\left(r_{2}-r_{1}\right), b_{3}(r)=2 /\left(r_{3}-r_{1}\right), b=\left(b_{1}, b_{2}, b_{3}\right)$. It is obvious that $b_{2}$ and $b_{3}$ define uniformly Lipschitz continuous functions from $\Delta_{\eta}$ to $C_{0}\left(\left[0, \varepsilon_{0}\right], \mathbb{R}\right)$. As for $b_{1}$, note that $r \mapsto 1 /\left(r_{3}-r_{1}\right)$ and $r \mapsto\left(r_{3}-r_{2}\right) /\left(r_{3}-r_{1}\right)$ are uniformly 
Lipschitz continuous on $\Delta_{\eta}$, and that for all $\varepsilon \in\left[0, \varepsilon_{0}\right], r \in \Delta_{\eta}$ :

$$
0 \leq \frac{r_{3}-r_{2}}{r_{3}-r_{1}}=1-\frac{r_{2}-r_{1}}{r_{3}-r_{1}} \leq 1-\frac{r_{2}-r_{1}}{r_{2}-r_{1}+\varepsilon} \leq 1-\frac{1}{1+\varepsilon / \eta} \leq \frac{\varepsilon_{0}}{\varepsilon_{0}+\eta}
$$

Since the function

$$
u \mapsto u(1-u)^{-4 / \kappa}\left(1-(1-u)^{1-4 / \kappa}\right)^{-1}
$$

extends to a $C^{1}$ function on [0,1[, it defines a uniformly Lipschitz continuous function on $\left[0, \varepsilon_{0} /\left(\varepsilon_{0}+\eta\right)\right]$. It follows that $b: \Delta_{\eta} \rightarrow C_{0}\left(\left[0, \varepsilon_{0}\right], \mathbb{R}^{3}\right)$ is uniformly Lipschitz continuous. So existence and strong unicity hold for the SDE

$$
d R_{t}=(\sqrt{\kappa}, 0,0) d \tilde{B}_{t}+b\left(R_{t}\right) d t,
$$

up to the first exit of $\Delta_{\eta}$. Since

$$
d\left(R_{3, t}-R_{2, t}\right)=-\frac{2\left(R_{3, t}-R_{2, t}\right) d t}{\left(R_{3, t}-R_{1, t}\right)\left(R_{3, t}-R_{1, t}\right)}
$$

the difference $\left(R_{3}-R_{2}\right)$ is decreasing, and a solution of the SDE is well defined until $\left(R_{2}-R_{1}\right)$ reaches $\eta$. Letting $\eta$ go to 0 , one gets existence and strong unicity up to explosion of $\left(R_{2}-R_{1}\right)^{-1}$.

Now, for any $\varepsilon \in\left(0, \varepsilon_{0}\right],\left(R_{t}(\varepsilon)\right)_{t}$ has the same law as $\left(W_{t}, g_{t}(z-\varepsilon), g_{t}(z)\right)_{t}$, where $\left(W_{t}\right)$ is the driving process of an $\mathrm{SLE}_{(0, y)}(\kappa, \kappa-4)$ conditionally on $Z \in$ $(z-\varepsilon, z)$, and $\left(g_{t}\right)$ are the corresponding conformal equivalences; for $\varepsilon=0$, one gets an $\operatorname{SLE}_{(0, z)}(\kappa,-4)$. So the driving process of an $\operatorname{SLE}_{(0, y)}(\kappa, \kappa-4)$ conditionally on $Z \in(z-\varepsilon, z)$ and stopped at time $\tau_{z-\varepsilon}$ converges in law as $\varepsilon \searrow 0$ to that of an $\operatorname{SLE}_{(0, z)}(\kappa,-4)$ stopped as time $\tau_{z}$ (after which it is no longer defined).

The future after $\tau_{z}$ is easier to handle. Indeed, from the Strong Markov property, an $\operatorname{SLE}_{(0, y)}(\kappa, \kappa-4)$ can be described as the concatenation of this process stopped at time $\tau_{z-\varepsilon}$ and an $\operatorname{SLE}_{\left(W_{\tau_{z-\varepsilon}}, g_{\tau_{z-\varepsilon}}(y)\right)}(\kappa, \kappa-4)$, independent of the former conditionally on its starting state. Conditioning by $Z \in(z-\varepsilon, z)$, one gets the concatenation of the original conditional process stopped at time $\tau_{z-\varepsilon}$ and an $\operatorname{SLE}(\kappa, \kappa-4)$ starting from $\left(W_{\tau_{z-\varepsilon}}, g_{\tau_{z-\varepsilon}}(z)\right)$ (as follows from (ii)), independent of the former conditionally on this starting state; note that $W_{\tau_{z-\varepsilon}} \leq$ $g_{\tau_{z-\varepsilon}}(z) \leq W_{\tau_{z-\varepsilon}}+\varepsilon$.

As $\varepsilon \searrow 0$, the conditional process up to time $\tau_{z-\varepsilon}$ converges to an $\operatorname{SLE}_{(0, z)}(\kappa,-4)$ stopped at time $\tau_{z}$, while the process after $\tau_{z-\varepsilon}$ converges in law to an $\operatorname{SLE}_{\left(W_{\tau_{z}}, W_{\tau_{z}}^{+}\right)}(\kappa, \kappa-$ 4 ), independent of the former conditionally on $W_{\tau_{z}}$. This concludes the proof.

We have mentioned that the $\operatorname{SLE}_{(0, y)}(\kappa, \kappa-4)$ could be seen as a standard $\operatorname{SLE}(\kappa)$ conditioned "never to swallow $y$ " (see [7, Section 5). Likewise, 
a $\operatorname{SLE}_{\left(0, y_{1}, y_{2}\right)}(\kappa, \kappa-4, \kappa-4), y_{1}<0<y_{2}$, can be interpreted as an $\operatorname{SLE}(\kappa)$ conditioned "never to swallow $y_{1}$ or $y_{2}$ ". On a heuristic level, this explains why $\operatorname{SLE}(\kappa, \kappa-4, \kappa-4)$ should have properties similar to those previously described for $\operatorname{SLE}(\kappa, \kappa-4)$.

Proposition 2. Let $\left(W_{t}, O_{t}^{1}, O_{t}^{2}\right)_{t}$ be the driving process of an $\operatorname{SLE}(\kappa, \kappa-4, \kappa-$ 4) starting from $\left(0, y_{1}, y_{2}\right), y_{1}<0<y_{2}$; let $\left(\mathcal{F}_{t}\right)$ the associated natural filtration. For some $x_{1} \in\left[y_{1}, 0\right), x_{2} \in\left(0, y_{2}\right]$, let $\tau_{i} \in(0, \infty]$ be the swallowing time of $x_{i}$, $i=1,2$.

(i) The following formula holds:

$$
\mathbb{P}\left(\tau_{1}=\infty, \tau_{2}=\infty\right)=\left(\frac{x_{1} x_{2}}{y_{1} y_{2}}\right)^{d}\left(\frac{x_{2}-x_{1}}{y_{2}-y_{1}}\right)^{\frac{\kappa}{2} d^{2}} .
$$

(ii) The law of the SLE conditionally on $\left\{\tau_{1}=\infty, \tau_{2}=\infty\right\}$ is that of an $\operatorname{SLE}(\kappa, \kappa-4, \kappa-4)$ starting from $\left(0, x_{1}, x_{2}\right)$.

(iii) Let $Z_{1}$ (resp. $Z_{2}$ ) be the leftmost (resp. rightmost) swallowed point of $\left(y_{1}, 0\right)$ (resp. $\left.\left(0, y_{2}\right)\right)$. Then $\left(Z_{1}, Z_{2}\right)$ is an $\mathcal{F}_{\infty}$-measurable r.v. with density:

$\mathbf{1}_{y_{1} \leq x_{1}<0<x_{2} \leq y_{2}} d^{2}\left(\frac{\kappa}{2}-1\right)\left(-\frac{1}{x_{1} x_{2}}+\frac{\kappa / 2-4}{\left(x_{2}-x_{1}\right)^{2}}\right)\left(\frac{x_{1} x_{2}}{y_{1} y_{2}}\right)^{d}\left(\frac{x_{2}-x_{1}}{y_{2}-y_{1}}\right)^{\frac{\kappa}{2} d^{2}} d x_{1} d x_{2}$.

Proof. (i) As in the previous proposition, there is only to check, using Itô's formula, that the following semimartingale

$$
\left(\frac{\left(g_{t}\left(x_{1}\right)-W_{t}\right)\left(g_{t}\left(x_{2}\right)-W_{t}\right)}{\left(g_{t}\left(y_{1}\right)-W_{t}\right)\left(g_{t}\left(y_{2}\right)-W_{t}\right)}\right)^{d}\left(\frac{g_{t}\left(x_{2}\right)-g_{t}\left(x_{1}\right)}{g_{t}\left(y_{2}\right)-g_{t}\left(y_{1}\right)}\right)^{\frac{\kappa}{2} d^{2}}
$$

is a local martingale.

(ii) Using (i) and Girsanov's theorem, one can give a proof that follows exactly that of 1 (ii). Note that if $\left(M_{t}\right)$ is the martingale considered in (i), and $\left(B_{t}\right)$ is the driving Brownian motion of the SLE, then:

$\frac{d\left\langle M_{t}, \sqrt{\kappa} B_{t}\right\rangle}{M_{t}}=(\kappa-4)\left(\left(\frac{1}{g_{t}\left(x_{1}\right)-W_{t}}+\frac{1}{g_{t}\left(x_{2}\right)-W_{t}}\right)-\left(\frac{1}{g_{t}\left(y_{1}\right)-W_{t}}+\frac{1}{g_{t}\left(y_{2}\right)-W_{t}}\right)\right) d t$

which is the difference between the drift term of an $\operatorname{SLE}_{\left(0, x_{1}, x_{2}\right)}(\kappa, \kappa-4, \kappa-4)$ and that of an $\operatorname{SLE}_{\left(0, y_{1}, y_{2}\right)}(\kappa, \kappa-4, \kappa-4)$. We shall give a clean interpretation of this identity later.

(iii) This follows directly from (i), since $\left\{x_{1}<Z_{1}<0<Z_{2}<x_{2}\right\}=\left\{\tau_{1}=\right.$ $\left.\tau_{2}=\infty\right\}$, from the definition of $Z_{1}, Z_{2}$.

\section{Frontier points of $\operatorname{SLE}(\kappa, \kappa-4)$ processes}

As before, $\kappa>4$ is fixed, and $d=1-\frac{4}{\kappa}$. Let $\left(W_{t}, O_{t}\right)_{t \geq 0}$ be the driving process of an $\operatorname{SLE}(\kappa, \kappa-4)$ starting from $\left(0,0^{+}\right) ;\left(K_{t}\right),\left(g_{t}\right),\left(\gamma_{t}\right)$ are as usual; 
$\left(\mathcal{F}_{t}\right)$ is the natural filtration of the Brownian motion driving the SLE. Then, if $Y_{t}=O_{t}-W_{t}, Y / \sqrt{\kappa}$ is a standard Bessel process of dimension $(2+d)$ starting from 0 . Indeed, it satisfies the SDE:

$$
d Y_{t}=d B_{t}+\frac{d+1}{2} d t
$$

where $B$ is the (standard) Brownian motion driving the SLE. For general background on Bessel processes, see [16, XI.1.

Let $t$ be a positive time, and $\sigma_{t}$ be the last time before $t$ when the tip of hull $\gamma_{t}$ was on the right-boundary of the whole hull $K_{\infty}$; this is obviously not a stopping time, and is analogous to the future infimum of a transient Bessel process for instance. Now define:

$$
X_{t}=g_{t}\left(\gamma_{\sigma_{t}}\right)-W_{t} .
$$

From the Markov property of SLE, $\left(g_{t}\left(K_{t+s} \backslash K_{t}\right)-W_{t}\right)_{s}$ defines an $\operatorname{SLE}_{\left(0, Y_{t}\right)}(\kappa, \kappa-$ 4), independent from $\mathcal{F}_{t}$ conditionally on $Y_{t}$. Then $X_{t}$ is the rightmost point on $\left(0, Y_{t}\right)$ swallowed by this SLE. It follows from scale invariance that $X_{t} / Y_{t}$ is independent from $\mathcal{F}_{t}$, and we have seen in Proposition 1 that $X_{t} / Y_{t} \stackrel{\mathcal{L}}{=} \operatorname{Beta}(d, 1)$.

As $X_{t}=Y_{t}\left(X_{t} / Y_{t}\right)$, these two variables being independent, one may work out the law of $X_{t}$. Indeed, for a standard Bessel process of dimension $\delta$ starting from 0 ,

$$
\mathbb{P}\left(\operatorname{BES}(\delta)_{t} \in d r\right)=\frac{2 r^{\delta-1} e^{-r^{2} / 2 t} d r}{\Gamma(\delta / 2)(2 t)^{\delta / 2}} .
$$

It follows that:

$$
\begin{aligned}
\mathbb{P}\left(X_{t} / \sqrt{\kappa} \in d x\right) & =\int_{x}^{\infty} \mathbb{P}\left(X_{t} / \sqrt{\kappa} \in d x \mid Y_{t} / \sqrt{\kappa} \in d y\right) \mathbb{P}\left(Y_{t} / \sqrt{\kappa} \in d y\right) \\
& =\int_{x}^{\infty} d \frac{x^{d-1} d x}{y^{d}} \cdot \frac{2 y^{d+1} e^{-y^{2} / 2 t}}{\Gamma(1+d / 2)(2 t)^{1+d / 2}} d y \\
& =\frac{d x^{d-1} e^{-x^{2} / 2 t}}{\Gamma(d / 2+1)(2 t)^{d / 2}} d x=\frac{2 x^{d-1} e^{-x^{2} / 2 t}}{\Gamma(d / 2)(2 t)^{d / 2}} d x
\end{aligned}
$$

So it turns out that $X_{t} / \sqrt{\kappa}$ is distributed like a standard $\operatorname{BES}(d)$ starting from 0 and taken at time $t$; taking squares, one may see this as a special case of beta-gamma algebra (see [5]). Let $\left(\mathcal{X}_{t}\right)$ be the filtration generated by this process. It appears readily that $\mathcal{X}_{t} \subset \mathcal{F}_{\infty}$, and $\mathcal{X}_{t} \nsubseteq \mathcal{F}_{s}$ for any $s \geq 0$.

The computation of the distribution of $X_{t}$ at a fixed time $t$ suggests the following generalization:

Proposition 3. The process $\left(X_{t} / \sqrt{\kappa}\right)_{t \geq 0}$ is a standard $\operatorname{BES}(d)$ process.

Proof. Let $\left(Q_{t}\right)$ designate the semigroup of a standard $d$-dimensional Bessel process. We will prove that for all $0 \leq s \leq t$, the law of $X_{t} / \sqrt{\kappa}$ conditionally 
on $\mathcal{X}_{s}$ is $Q_{t-s}\left(X_{s} / \sqrt{\kappa},.\right)$. The computation above shows that this holds for $0=s \leq t$.

Suppose now that $0<s<t$ are fixed. Almost surely, $X_{s}$ is positive. Conditionally on $\mathcal{X}_{s} \vee \mathcal{Y}_{s}$, the translated process $\left(Y_{s+u}\right)_{u}$ corresponds to an $\operatorname{SLE}_{\left(0, Y_{s}\right)}(\kappa, \kappa-4)$ conditioned on its rightmost swallowed point being $X_{s}$. Indeed, $\mathcal{X}_{s} \vee \mathcal{Y}_{s}=\sigma\left(\mathcal{Y}_{s}, X_{s}\right)$, since for $r \leq s, X_{r}=g_{r}\left(\gamma_{\sigma_{r}}\right)-W_{r}$, and:

$$
\sigma_{r}=\sup \left(u: u \leq r, \gamma_{u} \in g_{s}^{-1}\left(\left(\gamma_{\sigma_{s}}, \infty\right)\right)\right) \text {. }
$$

From Proposition 1 (iv), we know that $\left(X_{s+u}\right)_{u}$ (stopped on hitting 0) corresponds to an $\operatorname{SLE}_{\left(0, X_{s}\right)}(\kappa,-4)$ independent of $\mathcal{X}_{s} \vee \mathcal{Y}_{s}$ conditionally on $\left(Y_{s}, X_{s}\right)$. But $Y_{s}$ does not appear any longer in the conditional law (which essentially follows from Proposition [1 (ii)), so we have proved that the law $\left(X_{s+u} / \sqrt{\kappa}\right)_{u}$ stopped on hitting 0 conditionally on $\mathcal{X}_{s} \vee \mathcal{Y}_{s}$ is that of a standard $\operatorname{BES}(d)$ process starting from $X_{s} / \sqrt{\kappa}$, and stopped on hitting 0 .

Consider now $T$, an $\mathcal{X} \vee \mathcal{Y}$-stopping time a.s. supported on zeroes of $X$. We shall prove that $\left(g_{T+u} \circ g_{T}^{-1}\left(W_{T}^{+}\right)-W_{T+u}, X_{T+u}\right)_{u}$ is a copy of $\left(Y_{u}, X_{u}\right)_{u}$ independent from $(\mathcal{X} \vee \mathcal{Y})_{T}$. Indeed, let $0<t_{1}<t_{2}$; on the event $T \in\left(t_{1}, t_{2}\right), T$ is $(\mathcal{X} \vee \mathcal{Y})_{t_{2}}=\sigma\left(X_{t_{2}}, \mathcal{Y}_{t_{2}}\right)$-measurable. The process $\left(g_{t_{2}+u} \circ g_{t_{2}}^{-1}\left(X_{t_{2}}^{+}+W_{t_{2}}\right)-\right.$ $\left.W_{t_{2}+u}, X_{t_{2}+u}\right)_{u}$ is distributed as the process $\left(Y_{u}, X_{u}\right)$ for an $\operatorname{SLE}_{\left(0, X_{t_{2}}\right)}(\kappa, \kappa-4)$ conditioned on its right-most swallowed point being $X_{t_{2}}$, as described in Proposition 1(iv); this translated process is independent from $(\mathcal{X} \vee \mathcal{Y})_{t_{2}}$ conditionally on $X_{t_{2}}$. As $t_{1} \nearrow t_{2}$, since $X_{T}=0$ and $X$ is continuous, the law of the translated process converges to that of a copy of $\left(Y_{u}, X_{u}\right)_{u}$ independent from $(\mathcal{X} \vee \mathcal{Y})_{t_{2}}$. As this holds for any positive $t_{2}$, the claim follows.

There only remains to put things together. Let $0 \leq s \leq t$, and consider the $\mathcal{X} \vee \mathcal{Y}$-stopping time $T=\inf \left(u \in[s, t], X_{u}=0\right)$, with the convention $\inf \varnothing=\infty$. Let $\left(\tilde{X}_{u} / \sqrt{\kappa}\right)_{s \leq u \leq t}$ be a standard $\operatorname{BES}(d)$ process starting from $\tilde{X}_{s}=X_{s}$, and $\tilde{T}=\inf \left(u \in[s, t], \tilde{X}_{u}=0\right)$. Then the $[s, t] \cup\{\infty\}$-valued variables $T$ and $\tilde{T}$ have the same distribution. Either $T=\infty$, and $X_{t}$ is distributed as $\tilde{X}_{t}$ conditionally on $\tilde{T}=\infty$, or $T \leq t$; in the latter case, we have seen that $X_{t} / \sqrt{\kappa}$ is independent from $\sigma\left(\left(X_{u}\right)_{s \leq u \leq T}\right)$ conditionally on $T$, and has conditional distribution $Q_{t-T}(0,$.$) . From the Strong Markov property for Bessel$ processes, the following disintegration holds:

$$
Q_{t-s}(x, .)=\int_{s}^{t} Q_{t-u}(0, .) \mathbb{P}(\tilde{T} \in d u)+Q_{t-s}(x, . \mid \tilde{T}=\infty) \mathbb{P}(\tilde{T}=\infty)
$$

where $Q_{t-s}(x, . \mid \tilde{T}=\infty)$ designates the law of $\tilde{X}_{t}$ conditionally on $\inf _{s \leq u \leq t}\left(\tilde{X}_{u}\right)>$ 0 . Hence we have proved that (a regular version of) the distribution of $X_{t} / \sqrt{\kappa}$ conditionally on $(\mathcal{X} \vee \mathcal{Y})_{s}$ is $Q_{t-s}\left(X_{s} / \sqrt{\kappa},.\right)$, so that $\left(X_{t} / \sqrt{\kappa}\right)_{t}$ is a standard $d$-dimensional Bessel process.

We further discuss the relationship between the processes $X$ and $Y$. These two processes are Bessel processes of dimension $d$ and $(d+2)$ in their natural 
filtration; but these two filtrations differ, since $\mathcal{X}_{t} \not \subseteq \mathcal{Y}_{t}$. We will see later that in fact $\mathcal{Y}_{t} \subset \mathcal{X}_{t}$. Another important feature is the intertwining of these two Markov processes. Indeed, we have mentioned that $\left(X_{t} / Y_{t}\right)$ is distributed as a $\operatorname{Beta}(d, 1)$ variable, independently of $\mathcal{Y}_{t}$. So if $\left(P_{t}^{X}\right)$ and $\left(P_{t}^{Y}\right)$ denote the Markov semigroups of $X$ and $Y$ respectively (starting from 0 ), and $\Lambda$ is the Markov transition kernel specified by its action on bounded Borel functions on $\mathbb{R}^{+}$:

$$
(\Lambda f)(y)=d \int_{0}^{1} u^{d-1} f(u y) d u
$$

then the following intertwining relation holds: $P^{Y} \Lambda=\Lambda P^{X}$. Indeed, we have:

$$
\begin{aligned}
\mathbb{E}\left(f\left(X_{t}\right) \mid \mathcal{Y}_{s}\right) & =\mathbb{E}\left(f\left(X_{t}\right)\left|\mathcal{X}_{s} \vee \mathcal{Y}_{s}\right| \mathcal{Y}_{s}\right)=\mathbb{E}\left(P_{t-s}^{X} f\left(X_{s}\right) \mid \mathcal{Y}_{s}\right)=\left(\Lambda P^{X} f\right)\left(Y_{s}\right) \\
& =\mathbb{E}\left(f\left(X_{t}\right)\left|\mathcal{Y}_{t}\right| \mathcal{Y}_{s}\right)=\mathbb{E}\left((\Lambda f)\left(Y_{t}\right) \mid \mathcal{Y}_{s}\right)=\left(P^{Y} \Lambda f\right)\left(Y_{s}\right)
\end{aligned}
$$

as in [5], Proposition 2.1.

The definition and study of the process $X$ have been motivated by questions related to the final right boundary of the $\operatorname{SLE}(\kappa, \kappa-4)$ process. Notice that this boundary grows only when $X$ vanishes, so it is now natural to turn to the excursion theory of $X$ (away from 0 ). In particular the local time at 0 of $\left(X_{t}\right)$ will provide an adequate measure of the "size" of the right boundary.

Recall that for $\left(\rho_{t}\right)$ a Bessel process of dimension $\delta$, there exists a bicontinuous family of local times $\left(L_{t}^{a}\right)$ such that for any bounded Borel function $f$,

$$
\int_{0}^{t} f\left(\rho_{u}\right) d u=\int_{0}^{\infty} f(a) L_{t}^{a} a^{\delta-1} d a .
$$

(For Bessel local times, see e.g. [16, XI.1.25). Since $\rho$ has Brownian scaling, one gets:

$$
\left(L_{\lambda^{2} t}^{\lambda a}\right) \stackrel{\mathcal{L}}{=} \lambda^{2-\delta}\left(L_{t}^{a}\right)
$$

as a bicontinuous process of the two variables $t$ and $a$. As a consequence, if $\left(L_{t}^{0}\right)_{t \geq 0}$ is the local time at 0 of $\left(X_{t}\right)$, and if $\tau$. is its right-continuous inverse, then $\tau$. is a stable subordinator with index $\nu=1-\frac{d}{2}=\frac{1}{2}+\frac{2}{\kappa}$.

From the definition of $X$, it appears that the set of (global) frontier times of $\operatorname{SLE}(\kappa, \kappa-4)$ (i.e. times at which the trace lies on the right-boundary of the total hull $K_{\infty}$ ) is the zero set of $X$. Note that the a.s. Hausdorff dimension of frontier times has been derived in [1. Recall that the $\varphi$-measure of a set $Z$ is defined as:

$$
\varphi-m(Z)=\liminf _{\varepsilon>0}\left(\sum_{1}^{\infty} \varphi\left(\operatorname{diam}\left(J_{i}\right)\right), Z \subset \bigcup_{1}^{\infty} J_{i}, \operatorname{diam}\left(J_{i}\right)<\varepsilon\right) .
$$

Corollary 4. The set of frontier times of $\operatorname{SLE}(\kappa, \kappa-4)$ has a.s. Hausdorff dimension $(1 / 2+2 / \kappa)$. More precisely, the $\varphi_{\kappa}$-measure of frontier times is a.s. positive and locally finite, where:

$$
\varphi_{\kappa}(\varepsilon)=\varepsilon^{1 / 2+2 / \kappa}(\log |\log \varepsilon|)^{1 / 2-2 / \kappa} .
$$


Moreover, the $\varphi_{\kappa}$-measure of frontier times up to time $t$ is a multiple of the local time of $X$ at 0 .

Proof. The inverse of the local time of $X$ at 0 is a stable subordinator with index $(1 / 2+2 / \kappa)$. So the zero set of $X$ has the same distribution as the zero set of a stable Lévy process with index $(1 / 2-2 / \kappa)^{-1}$. Hence one can apply the results of [21].

We will also need some facts about principal values for $\delta \in(0,1)$, that we presently recall (see [2]). It is classical that one can consider a version of $\left(L_{t}^{a}\right)$ that is bicontinuous and Hölder in the space variable. More precisely, one can use the Biane-Yor identity (see [16]) to translate properties of Brownian local times (which are $(1 / 2-\varepsilon)$-Hölder in the space variable for any $\varepsilon>0)$ into results on Bessel local times. Hence one can define:

$$
p . v . \int_{0}^{t} \frac{d s}{\rho_{s}} \stackrel{\text { def }}{=} \int_{0}^{\infty}\left(L_{t}^{a}-L_{t}^{0}\right) a^{\delta-2} d a .
$$

As a function of $t$, this is continuous, and $C^{1}$ on $\left\{s: \rho_{s} \neq 0\right\}$, with derivative $1 / \rho_{s}$; though, it is not monotonic.

For $\delta>1, \rho$ is a semimartingale with decomposition:

$$
\rho_{t}=B_{t}+\frac{\delta-1}{2} \int_{0}^{t} \frac{d s}{\rho_{s}}
$$

where $B$ is a standard Brownian motion. If $0<\delta<1, \rho$ is no longer a semimartingale, but it still is a Dirichlet process (i.e. the sum of a squareintegrable local martingale and a process with zero quadratic variation):

$$
\rho_{t}=B_{t}+\frac{\delta-1}{2} p \cdot v \cdot \int_{0}^{t} \frac{d s}{\rho_{s}}
$$

where $B$ is a standard Brownian motion and the principal value has zero quadratic variation.

We turn back to the previous situation: $\left(W_{t}, O_{t}\right)$ is the driving mechanism of an $\operatorname{SLE}_{\left(0,0^{+}\right)}(\kappa, \kappa-4), d=1-4 / \kappa, Y_{t}=O_{t}-W_{t}, X_{t}=g_{t}\left(\gamma_{\sigma_{t}}\right)-W_{t}$.

Proposition 5. The following identity holds a.s.:

$$
W_{t}=-X_{t}+p \cdot v \cdot \int_{0}^{t} \frac{2 d s}{X_{s}}=-Y_{t}+\int_{0}^{t} \frac{2 d s}{Y_{s}} .
$$

Consequently, $\mathcal{Y}_{t}=\mathcal{F}_{t} \subset \mathcal{X}_{t}$ for all $t \geq 0$.

Proof. The second half of the identity is just the definition of $\operatorname{SLE}(\kappa, \kappa-4)$. A consequence of Loewner's equation and of the definition of $\left(X_{t}\right)$ is that:

$$
S_{t}=X_{t}+W_{t}-p \cdot v \cdot \int_{0}^{t} \frac{2 d s}{X_{s}}
$$


is a continuous process that is constant on the excursions of $X$ (recall that $X / \sqrt{\kappa}$ is a standard $d$-dimensional Bessel process). Indeed, $X_{t}=g_{t}\left(\gamma_{\sigma_{t}}\right)-W_{t}$, and $\sigma$. is constant on the excursions of $X$. Define $\tilde{S}_{u}=S_{\tau_{u}}$ where $\tau_{u}$ is the rightcontinuous inverse of $\left(L_{t}^{0}\right)$, the local time at 0 of $X$. Since $\left(S_{t}\right)$ is continuous and constant on the excursions of $X$, the process $\tilde{S}$ has a continuous version. Indeed, $\tilde{S}$ is càdlàg; suppose that is has a discontinuity at $u$. Necessarily, $\tau_{u}>\tau_{u}^{-}$, and $\left(\tau_{u}^{-}, \tau_{u}\right)$ is an excursion interval for $X$, so $S_{\tau_{u}^{-}}=S_{\tau_{u}}$. Since the local time at 0 of $X$ is instantaneously increasing at the right of $\tau_{u}$ and at the left of $\tau_{u}^{-}$, and $S$ is continuous at $\tau_{u}$ and $\tau_{u}^{-}$, so is $\tilde{S}$.

Moreover, for $u>0, \tau_{u}$ is a stopping time for $X$ such that $X_{\tau_{u}}=0$ a.s. . As we have seen in the proof of Proposition 3 this implies that $\left(X_{\tau_{u}+t}, W_{\tau_{u}+t}-\right.$ $\left.W_{\tau_{u}}\right)_{t}$ has the same law as $\left(X_{t}, W_{t}\right)_{t}$ and is independent from $\mathcal{X}_{\tau_{u}} \vee \mathcal{F}_{\tau_{u}}$. From the definition of $S$ and $\tilde{S}$, it appears that $\tilde{S}_{u+v}=\tilde{S}_{u}+\tilde{S}_{v}^{\prime}$ where $\tilde{S}^{\prime}$ is a copy of $\tilde{S}$ independent from $\sigma\left(\left(X_{s}, W_{s}\right)_{s \leq \tau_{u}}\right)$. So $\tilde{S}$ is a continuous Lévy process, i.e. a Brownian motion with drift. But $S$ has Brownian scaling, and $\tau$. has index $\nu=1-d / 2$, so $\tilde{S}$ has index $2 \nu \in(1,2)$. Hence $\tilde{S}$ and $S$ vanish identically, which proves the first half of the identity.

Furthermore, $W_{t}$ is $\mathcal{X}_{t}$ measurable for all $t$, so $\mathcal{Y}_{t}=\mathcal{F}_{t} \subset \mathcal{X}_{t}$ for all $t \geq 0$.

Before proceeding with the study of $\operatorname{SLE}(\kappa, \kappa-4)$, we sum up some of these results in an SLE-free formulation.

Corollary 6. For $d \in(0,1)$, let $\rho_{1}$ (resp. $\left.\rho_{2}\right)$ be a standard Bessel process of dimension $d$ (resp. $(d+2)$ ), and $\mathcal{F}^{1}$ (resp. $\left.\mathcal{F}^{2}\right)$ be its natural filtration. Then there exists a coupling of $\rho_{1}$ and $\rho_{2}$ such that:

(i) $\mathcal{F}_{t}^{2} \subset \mathcal{F}_{t}^{1}$ for all $t \geq 0$.

(ii) The process $\left(\rho_{2}-\rho_{1}\right)$ has zero quadratic variation.

(iii) The semigroups of $\rho_{1}, \rho_{2}$ are intertwined by multiplication by a $\operatorname{Beta}(d, 1)$

law. More precisely, if $f$ is some bounded Borel function on $\mathbb{R}^{+}$, then:

$$
\mathbb{E}\left(f\left(\rho_{1, t}\right) \mid \mathcal{F}_{t}^{2}\right)=\int_{0}^{1} f\left(u \rho_{2, t}\right) d u^{d-1} d u .
$$

(iv) The following identity holds a.s. for all $t \geq 0$ :

$$
\rho_{1, t}+\frac{d-1}{2} p \cdot v \cdot \int_{0}^{t} \frac{d s}{\rho_{1, s}}=\rho_{2, t}+\frac{d-1}{2} \int_{0}^{t} \frac{d s}{\rho_{2, s}} .
$$

(v) Let $\rho_{1, t}=B_{t}^{1}+\frac{d-1}{2} p \cdot v \cdot \int_{0}^{t} \frac{d s}{\rho_{1, s}}$ and $\rho_{2, t}=B_{t}^{2}+\frac{d+1}{2} \int_{0}^{t} \frac{d s}{\rho_{2, s}}$ be the Dirichlet process decompositions of $\rho_{1}$ and $\rho_{2}$. Then:

$$
\begin{aligned}
B_{t}^{2} & =\mathbb{E}\left(B_{t}^{1} \mid \mathcal{F}_{t}^{2}\right) \\
d \int_{0}^{t} \frac{d s}{\rho_{2, s}} & =(d-1) \mathbb{E}\left(p . v \cdot \int_{0}^{t} \frac{d s}{\rho_{1, s}} \mid \mathcal{F}_{t}^{2}\right) .
\end{aligned}
$$


Proof. With $d=1-4 / \kappa$, define $\rho_{1}=X / \sqrt{\kappa}$ and $\rho_{2}=Y / \sqrt{\kappa}$, where $X$ and $Y$ are as above. We have already proved (i), (iii), (iv). For (ii), note that:

$$
\sqrt{\kappa}\left(\rho_{2, t}-\rho_{1, t}\right)=Y_{t}-X_{t}=p \cdot v \cdot \int_{0}^{t} \frac{2 d s}{X_{s}}-\int_{0}^{t} \frac{2 d s}{Y_{s}} .
$$

(v) Taking conditional expectations in (iv), one gets:

$$
\mathbb{E}\left(\rho_{1, t}+\frac{d-1}{2} p \cdot v \cdot \int_{0}^{t} \frac{d s}{\rho_{1, s}} \mid \mathcal{F}_{t}^{2}\right)=\rho_{2, t}+\frac{d-1}{2} \int_{0}^{t} \frac{d s}{\rho_{2, s}}
$$

since the right-hand side is $\mathcal{F}_{t}^{2}$-measurable. It follows that:

$$
\mathbb{E}\left(B_{t}^{1} \mid \mathcal{F}_{t}^{2}\right)-B_{t}^{2}=d \int_{0}^{t} \frac{d s}{\rho_{2, s}}-(d-1) \mathbb{E}\left(p . v \cdot \int_{0}^{t} \frac{d s}{\rho_{1, s}} \mid \mathcal{F}_{t}^{2}\right) .
$$

The left-hand side is a continuous square-integrable $\mathcal{F}^{2}$ - martingale, while the right-hand side has zero quadratic variation; so both sides vanish identically.

Taking expectations in (v), one gets:

$$
d \mathbb{E}\left(\int_{0}^{t} \frac{d s}{\rho_{2, s}}\right)=(d-1) \mathbb{E}\left(p \cdot v \cdot \int_{0}^{t} \frac{d s}{\rho_{1, s}}\right) .
$$

Because of Brownian scaling, the two sides are proportional to $t \mapsto \sqrt{t}$. One can check directly that the coefficients agree. Indeed, from the explicit distribution of $\rho_{1, t}, \rho_{2, t}$ (starting from 0 ), it is easy to compute:

$$
\mathbb{E}\left(\rho_{1, t}\right)=\sqrt{2 t} \frac{\Gamma\left(\frac{d+1}{2}\right)}{\Gamma\left(\frac{d}{2}\right)}, \quad \mathbb{E}\left(\rho_{2, t}\right)=\sqrt{2 t} \frac{\Gamma\left(\frac{d+3}{2}\right)}{\Gamma\left(\frac{d+2}{2}\right)} .
$$

From the Dirichlet decompositions, one gets:

$$
\begin{array}{r}
(d-1) \mathbb{E}\left(p . v \cdot \int_{0}^{t} \frac{d s}{\rho_{1, s}}\right)=2 \mathbb{E}\left(\rho_{1, t}\right)=2 \frac{\Gamma\left(\frac{d+1}{2}\right)}{\Gamma\left(\frac{d}{2}\right)} \sqrt{2 t} \\
d \mathbb{E}\left(\int_{0}^{t} \frac{d s}{\rho_{2, s}}\right)=\frac{2 d}{d+1} \mathbb{E}\left(\rho_{2, t}\right)=\frac{2 d}{d+1} \cdot \frac{\Gamma\left(\frac{d+3}{2}\right)}{\Gamma\left(\frac{d+2}{2}\right)} \sqrt{2 t}=2 \frac{\Gamma\left(\frac{d+1}{2}\right)}{\Gamma\left(\frac{d}{2}\right)} \sqrt{2 t} .
\end{array}
$$

In the limiting case $d \nearrow 1, \rho_{1}$ is a reflecting Brownian motion that can be represented as: $\left|B_{t}\right|=\tilde{B}_{t}+\ell_{t}^{0}$, where $\ell^{0}$ is the local time at 0 of the Brownian motion $B$ and $\tilde{B}$ is also a Brownian motion. Assuming that the Dirichlet decomposition of $\rho_{1}$, i.e. $\left(B_{t}^{1}, \frac{d-1}{2} \int_{0}^{t} d s / \rho_{1, s}\right)$ converges to the Dirichlet (or semimartingale) decomposition of $|B|$, that is $\left(\tilde{B}_{t}, \ell_{t}^{0}\right)$, then (iv) translates into:

$$
\tilde{B}_{t}+2 \ell_{t}^{0}=\rho_{2, t}
$$


where $\rho_{2}$ is a 3 -dimensional Bessel process; this is Pitman's $(2 M-X)$ theorem. One also gets the intertwining relation

$$
\mathbb{E}\left(f\left(\rho_{1, t}\right) \mid \mathcal{F}_{t}^{2}\right)=\int_{0}^{1} f\left(u \rho_{2, t}\right) d u
$$

which is used in Pitman's original proof.

Let us get back to $\operatorname{SLE}(\kappa, \kappa-4)$. In the discussion in 7 , Section 5 , we mentioned that the future of an $\operatorname{SLE}(\kappa, \kappa-4)$ after a random time where the trace lies on the (final) right boundary is a translated $\operatorname{SLE}_{\left(0,0^{+}\right)}(\kappa, \kappa-4)$. Since the trace lies on the right boundary exactly when the process $X$ vanish, a rigorous statement and proof were given in the proof of Proposition 3 In particular, we consider the stopping times $\tau_{u}, u \geq 0$, where $\tau$ is the right-continuous inverse of the local time of $X$ at 0 . These are $\mathcal{X}$-stopping times a.s. supported on the zero set of $X$, so that if $\left(K_{t}\right),\left(g_{t}\right)$ denote the families of hulls and conformal equivalences respectively, then for any $u \geq 0,\left(g_{\tau_{u}}\left(K_{\tau_{u}+t} \backslash K_{\tau_{u}}\right)-W_{\tau_{u}}\right)_{t}$ defines an $\operatorname{SLE}_{\left(0,0^{+}\right)}(\kappa, \kappa-4)$ independent from $K_{\tau_{u}}$.

Let $\mathcal{Q}$ be the set of bounded hulls in $\mathbb{H}$; recall that a bounded hull $A$ in $\mathbb{H}$ is a compact subset of $\overline{\mathbb{H}}$ such that $\mathbb{H} \backslash A$ is simply connected and $A \cap \mathbb{R} \subset \overline{A \cap \mathbb{H}}$. Then $\mathcal{Q} \times \mathbb{R}$ can be seen as a semigroup of "pointed hulls" with composition law:

$$
(A, x) \cdot(B, y)=\left(A \cup \phi_{A}^{-1}(B+x), x+y\right)
$$

Note that $(A, x) \mapsto(\operatorname{cap}(A), x)$, where cap denotes the half-plane capacity, is a semigroup morphism $\mathcal{Q} \times \mathbb{R} \rightarrow \mathbb{R}^{+} \times \mathbb{R}$. The only invertible elements in $\mathcal{Q} \times \mathbb{R}$ are the $(\varnothing, x), x \in \mathbb{R}$.

Consider now the $\mathcal{Q} \times \mathbb{R}$-valued process: $u \mapsto\left(K_{\tau_{u}}, W_{\tau_{u}}\right)$. Then this process has the independent increment property; this follows readily from the fact that $\left(g_{\tau_{u}}\left(K_{\tau_{u}+t} \backslash K_{\tau_{u}}\right)-W_{\tau_{u}}\right)_{t}$ defines an $\operatorname{SLE}_{\left(0,0^{+}\right)}(\kappa, \kappa-4)$ independent from $K_{\tau_{u}}$. Taking into account the restriction formulae discussed in the previous chapter, one can formulate the:

Proposition 7. Let $H_{u}=K_{\tau_{u}}, w_{u}=W_{\tau_{u}}$. Then $\left(H_{u}, w_{u}\right)$ is a $\mathcal{Q} \times \mathbb{R}$-valued stable Lévy process with index $\nu=\frac{1}{2}+\frac{2}{\kappa}$ in the following sense:

(i) For any $u, v \geq 0$, if $(\tilde{H}, \tilde{w})$ is an independent copy of $(H, w)$, then:

$$
\left(H_{u}, w_{u}\right) \cdot\left(\tilde{H}_{v}, \tilde{w}_{v}\right) \stackrel{\mathcal{L}}{=}\left(H_{u+v}, w_{u+v}\right) .
$$

(ii) For any $c>0, u \geq 0,\left(H_{c u}, w_{c u}\right)_{u} \stackrel{\mathcal{L}}{=} c^{1 / 2 \nu}\left(H_{u}, w_{u}\right)_{u}$.

Moreover, $\left(H_{u}, w_{u}\right)$ satisfies the following restriction formula: for any smooth + -hull $A$, if $\alpha=\frac{1}{2}-\frac{1}{\kappa}$, and $L$ is an independent loop soup with intensity $\lambda_{\kappa}$, then:

$$
\phi_{A}^{\prime}(0)^{\alpha}=\mathbb{E}\left(\phi_{\phi_{H_{u}}(A)}^{\prime}\left(w_{u}\right)^{\alpha} \mathbf{1}_{H_{u} \cap A^{L}=\varnothing}\right) .
$$

Obviously, the image of a semigroup-valued Lévy process under a semigroup homomorphism is again a Lévy process. Of particular interest is the $\mathbb{R}^{+} \times \mathbb{R}$ - 
valued process:

$$
\left(\tau_{u}, w_{u}\right)_{u}=\left(\tau_{u}, p \cdot v \cdot \int_{0}^{\tau_{u}} \frac{2 d s}{X_{s}}\right)
$$

where the second coordinate is a well-known stable Lévy process with index $2 \nu=1+4 / \kappa$.

We now describe the excursion process for these excursions "away from the right-boundary". Consider the space $U$ of real continuous processes with finite lifetime (with the usual topology): an element $w$ of $U$ defines a continuous realvalued function on $[0, T(w)]$, where $T(w)>0$ is the lifetime of $w$. Then one can define a Loewner map from a subset of $U$ to $\mathcal{Q} \times \mathbb{R}$ in the following way: for a suitable $w \in U$, with lifetime $T>0$, let $\left(\tilde{g}_{t}\right)$ be the Loewner flow associated with the driving function $w$, and $\left(\tilde{K}_{t}\right)$ the corresponding hulls. Then $\mathbf{L} w=\left(\tilde{K}_{T}, w_{T}\right)$ defines an element of $\mathcal{Q} \times \mathbb{R}$.

We recall a representation of excursions of Bessel processes (see e.g. 15; see also 3 ). The excursion measure $n_{d}$ of a $d$-dimensional Bessel process, $0<d<2$, seen as a measure on $U$, can be described as follows (up to a multiplicative constant):

- The maximum $M$ of the excursion satisfies $n_{d}(M>x)=x^{d-2}$ for $x>0$.

- Under the probability measure $n_{d}\left(. \mathbf{1}_{M>x}\right) / n_{d}(M>x)$, the excursion is the concatenation of a $(4-d)$-dimensional Bessel process, run until it hits the level $x$, and an independent $\operatorname{BES}(d)$ process started from $x$, killed when it hits 0 .

We briefly justify this decomposition. If $T_{x}$ is the first time at which the $d$-dimensional Bessel process $\rho$ (started from 0 ) reaches the level $x$, consider the excursion straddling $T_{x}$. From the Poisson process property, this excursion has distribution $n_{d}(. \mid M \geq x)$; so the only thing to check is that $\left(\rho_{u}\right)_{g_{T_{x}} \leq u \leq T_{x}}$ is a $\operatorname{BES}(4-d)$ stopped when it hits $x$. This follows from the realization of the $\operatorname{BES}(4-d)$ process as a Doob $h$-transform of the $\operatorname{BES}(d)$ process.

This decomposition translates into a description of the excursions "away from the boundary" for $\operatorname{SLE}(\kappa, \kappa-4)$. We retain the previous notations.

Proposition 8. The process $\left(e_{u}\right)_{u}$ defined by $e_{u}=\left(g_{\tau_{u}-}^{-1}\left(K_{\tau_{u}} \backslash K_{\tau_{u^{-}}}\right), W_{\tau_{u}}-\right.$ $\left.W_{\tau_{u^{-}}}\right)$if $\tau_{u}>\tau_{u^{-}}$, and by $e_{u}=\partial$ if $\tau_{u}=\tau_{u^{-}}$, is $a(\mathcal{Q} \times \mathbb{R}) \cup\{\partial\}$-valued $\left(\mathcal{X}_{\tau_{u}}\right)$ Poisson point process. Its characteristic measure $N_{\kappa}$ is the image of $n_{d}$ under the composition:

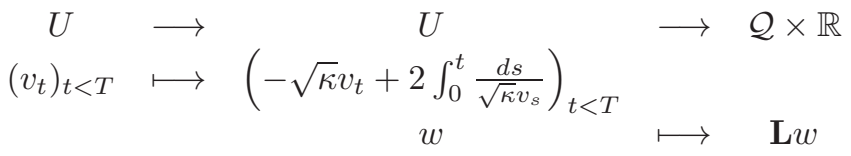

(with the natural extension for cemetary states). In other words, the excursion measure $N_{\kappa}$ can be described (up to a multiplicative constant) as the monotone 
limit of the measures $y^{d-2} N_{\kappa, y}$ as $y \searrow 0$, where $N_{\kappa, y}$ is the probability measure resulting from the concatenation of:

- an $\operatorname{SLE}_{\left(0,0^{+}\right)}(\kappa, \kappa)$, with associated driving process $\hat{W}$ and conformal equivalences $\hat{g}_{t}$, run until $\left(\hat{g}_{t}\left(0^{+}\right)-\hat{W}_{t}\right)$ hits y at time $\hat{T}$

- and an independent (conditionally on its initial state) $\operatorname{SLE}_{\left(\hat{W}_{\hat{T}}, \hat{g}_{\hat{T}}\left(0^{+}\right)\right)}(\kappa,-4)$ with associated driving process $\tilde{W}$ and conformal equivalences $\tilde{g}_{t}$, run until $\left(\tilde{g}_{t}\left(\hat{W}_{\hat{T}}+y\right)-\tilde{W}_{t}\right)$ hits 0.

Recall the notations of [7, Section 6. A (somewhat wishful) roadmap to proving duality would involve:

- Computing the tails of the distribution of the first two moments (i.e. translation at infinity and capacity seen from infinity) of the "synthetic" hull $K_{2}: \phi_{K_{2}}(z)=z-w+2 \tau / z+O\left(1 / z^{2}\right)$,

- Proving the convergence in distribution:

$$
\left(\frac{\tau^{(1)}+\cdots+\tau^{(n)}}{n^{1 / \nu}}, \frac{w^{(1)}+\cdots+w^{(n)}}{n^{1 / 2 \nu}}\right) \longrightarrow\left(\tau_{l}, \int_{0}^{\tau_{l}} 2 d s / X_{s}\right)
$$

where $\left(\tau^{(i)}, w^{(i)}\right)$ are the first two moments of an independent copy $K_{2}^{(i)}$ of $K_{2}$ and $l$ is some constant,

- Proving that a stable law on hulls is determined by the joint law of its first two moments under suitable conditions, and

- Proving that the right boundary of the concatenation $K_{2}^{(1)} \ldots K_{2}^{(n)}$ is an $\operatorname{SLE}_{l}\left(\kappa^{\prime}, \kappa^{\prime} / 2-2\right)$ stopped at a random time.

\section{Cutpoints for $\operatorname{SLE}(\kappa, \kappa-4, \kappa-4)$}

In the previous section, we were able to explicit the different processes involved, since they were $\mathbb{R}^{+}$-valued diffusions satisfying Brownian scaling, hence Bessel processes. In the two-sided case we are now discussing, one has to recast the arguments in a more abstract fashion, that also sheds new light on the one-sided case studied in the previous section. We begin with a rephrasing of the previous construction, based on Doob $h$-transforms and related Girsanov transformations (see [25], where Girsanov densities are interpreted in terms of restriction measures).

As before, $\kappa>4$ is fixed, $d=1-4 / \kappa ;\left(W_{t}\right)$ is the Brownian motion driving an $\operatorname{SLE}_{0}(\kappa)$ process, and $g_{t}$ are the associated conformal equivalences. The notations $\mathbb{P}$ and $\mathbb{E}$ refer to this process. 
For any $y>0, Y_{t}=g_{t}(y)-W_{t}$ defines a $\operatorname{BES}(2-d)$ process; as is well known, $h(u)=u^{d}$ is a scale function for this diffusion. Hence, for any $y>0$ and $t>0$, if $\tau_{y}$ denotes the swallowing time of $y$ and $Y_{t}=g_{t}(y)-W_{t}$, then:

$$
\left(P_{t}^{\uparrow} f\right)(y)=\frac{1}{h(y)} \mathbb{E}\left(f\left(Y_{t}\right) \mathbf{1}_{\tau_{y}>t} h\left(Y_{t}\right)\right)
$$

defines a Markov semigroup $\left(\left(\mathbf{1}_{\tau_{y}>t} h\left(Y_{t}\right)\right)_{t}\right.$ is an $L^{1}$ - martingale under $\left.\mathbb{P}\right)$. Note that the probability measures $P_{t}(y,$.$) admit a weak limit as y \searrow 0$. The infinitesimal generator of this semigroup is obtained by conjugation of the original generator, and it is easily seen that $\left(P_{t}^{\uparrow}\right)$ is the semigroup of $\left(Y_{t}=g_{t}(y)-W_{t}\right)$ under the $\operatorname{SLE}(\kappa, \kappa-4)$ probability measure. Let $\mathbb{P}^{\uparrow}$ be a corresponding probability measure (i.e. under $\mathbb{P}^{\uparrow}, Y / \sqrt{\kappa}$ is a $\mathrm{BES}(2+d)$ process). In terms of Bessel processes, this is a classical result, see e.g. [15].

Now, let $0<x<y, Y_{t}=g_{t}(y)-W_{t}$ as above, and $X_{t}=g_{t}(x)-W_{t}$. We look at $\left(X_{t}\right)$ as a functional of $\left(Y_{t}\right)$, as a consequence of the identity:

$$
d\left(X_{t}-Y_{t}\right)=-\frac{2\left(X_{t}-Y_{t}\right) d t}{X_{t} Y_{t}} .
$$

Then the semimartingale $\left(\mathbf{1}_{t<\tau_{x}} h\left(X_{t}\right) / h\left(Y_{t}\right)\right)$ is a $\mathbb{P}^{\uparrow}$-martingale. Indeed

$$
\frac{1}{h(y)} \mathbb{E}\left(\left(\frac{h\left(X_{t}\right)}{h\left(Y_{t}\right)} \mathbf{1}_{\tau_{x}>t}\right) \mathbf{1}_{\tau_{y}>t} h\left(Y_{t}\right)\right)=\frac{1}{h(y)} \mathbb{E}\left(\mathbf{1}_{\tau_{x}>t} h\left(X_{t}\right)\right)=\frac{h(x)}{h(y)} .
$$

Furthermore, $h(x) / h(y)=\mathbb{P}^{\uparrow}\left(\tau_{x}=\infty\right)$, so one gets for any bounded Borel function $f$ :

$\mathbb{E}_{y}^{\uparrow}\left(f\left(X_{t}\right) \mid \tau_{x}=\infty\right)=\frac{h(y)}{h(x)} \cdot \frac{1}{h(y)} \mathbb{E}\left(f\left(X_{t}\right)\left(\frac{h\left(X_{t}\right)}{h\left(Y_{t}\right)} \mathbf{1}_{\tau_{x}>t}\right) \mathbf{1}_{\tau_{y}>t} h\left(Y_{t}\right)\right)=P_{t}^{\uparrow} f(x)$,

which is essentially Proposition 1 (i)-(ii). This is formally very similar to the following properties of the three-dimensional Bessel process: the BES(3) process is the Doob $h$-transform of a Brownian motion, with $h(x)=x$; if $\rho$ is a $\operatorname{BES}(3)$ process, then conditionally on $\inf _{s \geq 0}\left(\rho_{s}\right) \geq a, \rho-a$ is again a $\operatorname{BES}(3)$ process.

We have also used a description of $\operatorname{SLE}(\kappa, \kappa-4)$ conditionally on $Z$, its rightmost swallowed point. In the present set-up, note that $1-h(x) / h(y)=$ $\mathbb{P}^{\uparrow}\left(\tau_{x}<\infty\right)$, so:

$$
\begin{aligned}
\mathbb{E}_{y}^{\uparrow}\left(f\left(X_{t}\right) \mathbf{1}_{\tau_{x}>t} \mid \tau_{x}<\infty\right) & =\left(1-\frac{h(x)}{h(y)}\right)^{-1} \frac{1}{h(y)} \mathbb{E}\left(f\left(X_{t}\right) \mathbf{1}_{\tau_{x}>t}\left(1-\frac{h\left(X_{t}\right)}{h\left(Y_{t}\right)}\right) \mathbf{1}_{\tau_{y}>t} h\left(Y_{t}\right)\right) \\
& =\frac{1}{h(y)-h(x)} \mathbb{E}\left(f\left(X_{t}\right) \mathbf{1}_{\tau_{x}>t}\left(h\left(Y_{t}\right)-h\left(X_{t}\right)\right)\right) .
\end{aligned}
$$

Now let $x \nearrow y$ (a rigorous proof of these results has already been given, so we can afford to argue a bit loosely here). One gets:

$$
\mathbb{E}_{y}^{\uparrow}\left(f\left(Y_{t}\right) \mathbf{1}_{\tau_{y}>t} \mid Z \in d y\right)=\frac{1}{h^{\prime}(y)} \mathbb{E}\left(f\left(Y_{t}\right) \mathbf{1}_{\tau_{y}>t} g_{t}^{\prime}(y) h^{\prime}\left(Y_{t}\right)\right) d y .
$$


It is indeed easy to check that $\left(g_{t}^{\prime}(y)\left(g_{t}(y)-W_{t}\right)^{d-1} \mathbf{1}_{\tau_{y} \geq t}\right)_{t}$ is an integrable $\mathbb{P}$-local martingale. So we can define a (non conservative) Markov semigroup:

$$
\left(P_{t}^{\downarrow} f\right)(y)=\frac{1}{h^{\prime}(y)} \mathbb{E}\left(f\left(Y_{t}\right) \mathbf{1}_{\tau_{y}>t} g_{t}^{\prime}(y) h^{\prime}\left(Y_{t}\right)\right)
$$

which corresponds to an $\operatorname{SLE}_{(0, y)}(\kappa,-4)$ killed at $\tau_{y}$.

Finally, the excursion measure of $X$ can be described in terms of BES $(4-d)$ and $\operatorname{BES}(d)$ processes. The $\operatorname{BES}(d)$ process corresponds to the semigroup $P^{\downarrow}$, while the $\operatorname{BES}(4-d)$ process corresponds to the semigroup $P^{\uparrow}$, where:

$$
\left(P_{t}^{\uparrow} f\right)(y)=\frac{1}{y} \mathbb{E}\left(f\left(Y_{t}\right) \mathbf{1}_{\tau_{y}>t} g_{t}^{\prime}(y) Y_{t}\right) .
$$

To sum up, we have encountered $\operatorname{SLE}(\kappa, \rho)$ processes with $\rho=0, \kappa-4,-4, \kappa$, associated with Bessel processes of dimensions $\delta=2-d, 2+d, d, 4-d$ (or index $\nu=-1 / 2+2 / \kappa, 1 / 2-2 / \kappa,-1 / 2-2 / \kappa, 1 / 2+2 / \kappa)$; the corresponding semigroups are $P, P^{\uparrow}, P^{\downarrow}, P^{\uparrow}$, defined by means of the $\mathbb{P}$ - local martingales $(\mathbf{1}),\left(Y_{t}^{d}\right),\left(g_{t}^{\prime}(y) Y_{t}^{d-1}\right),\left(g_{t}^{\prime}(y) Y_{t}\right)$. Note that these densities are also considered in 25$]$.

We now turn to the two-sided case. Under $\mathbb{P}, W / \sqrt{\kappa}$ is a standard Brownian motion, $g_{t}$ are the associated conformal equivalences, and $\mathcal{F}$ is the natural filtration. If $y_{1}<0<y_{2}$, then it is easy to check that:

$$
\left(W_{t}-g_{t}\left(y_{1}\right)\right)^{d}\left(g_{t}\left(y_{2}\right)-W_{t}\right)^{d}\left(g_{t}\left(y_{2}\right)-g_{t}\left(y_{1}\right)\right)^{\frac{\kappa}{2} d^{2}} \mathbf{1}_{\tau_{y_{1}}>t, \tau_{y_{2}}>t}
$$

is a martingale. Let $k\left(u_{1}, u_{2}\right)=\left(-u_{1}\right)^{d} u_{2}^{d}\left(u_{2}-u_{1}\right)^{\kappa d^{2} / 2}$, and define:

$$
\left(Q_{t}^{\uparrow \uparrow} f\right)\left(y_{1}, y_{2}\right)=\frac{1}{k\left(y_{1}, y_{2}\right)} \mathbb{E}\left((f k)\left(g_{t}\left(y_{1}\right)-W_{t}, g_{t}\left(y_{2}\right)-W_{t}\right) \mathbf{1}_{\tau_{y_{1}}>t, \tau_{y_{2}}>t}\right)
$$

for any bounded Borel function $f$ on $\mathbb{R}^{-} \times \mathbb{R}^{+}$. As before, this is the semigroup of an $\left(\mathbb{R}^{+}\right)^{2}$-valued Markov process. If $\mathcal{L}$ and $\mathcal{L}^{\uparrow \uparrow}$ designate the infinitesimal generator of the diffusion $\left(g_{t}\left(y_{1}\right)-W_{t}, g_{t}\left(y_{2}\right)-W_{t}\right)$ under $\mathbb{P}$ and the generator of $Q^{\uparrow \uparrow}$ respectively, say restricted to $C_{0}^{2}\left(\mathbb{R}^{-} \times \mathbb{R}^{+}\right)$, then:

$$
\begin{aligned}
\mathcal{L} & =\frac{\kappa}{2}\left(\frac{\partial}{\partial u_{1}}+\frac{\partial}{\partial u_{2}}\right)^{2}+\frac{2}{u_{1}} \frac{\partial}{\partial u_{1}}+\frac{2}{u_{2}} \frac{\partial}{\partial u_{1}} \\
\mathcal{L}^{\uparrow \uparrow} & =k\left(u_{1}, u_{2}\right)^{-1} \mathcal{L} k\left(u_{1}, u_{2}\right) \\
& =\frac{\kappa}{2}\left(\frac{\partial}{\partial u_{1}}+\frac{\partial}{\partial u_{2}}\right)^{2}+\left(\frac{\kappa-2}{u_{1}}+\frac{\kappa-4}{u_{2}}\right) \frac{\partial}{\partial u_{1}}+\left(\frac{\kappa-4}{u_{1}}+\frac{\kappa-2}{u_{2}}\right) \frac{\partial}{\partial u_{2}}
\end{aligned}
$$

where $k$ is seen as a multiplication operator. So $\mathcal{L}^{\uparrow \uparrow}$ is the generator of an $\operatorname{SLE}(\kappa, \kappa-4, \kappa-4)$, and we have identified $Q^{\uparrow \uparrow}$. If $y_{1}<x_{1}<0<x_{2}<y_{2}$, it now appears that

$$
\frac{k\left(g_{t}\left(x_{1}\right)-W_{t}, g_{t}\left(x_{2}\right)-W_{t}\right)}{k\left(g_{t}\left(y_{1}\right)-W_{t}, g_{t}\left(y_{2}\right)-W_{t}\right)} \mathbf{1}_{\tau_{x_{1}}>t, \tau_{x_{2}}>t}
$$


is a martingale under an $\operatorname{SLE}_{\left(0, y_{1}, y_{2}\right)}(\kappa, \kappa-4, \kappa-4)$ probability measure, which is Proposition 2 (i). Proposition 2 (ii) also follows immediately.

We now discuss the two-sided analogue of Proposition 1(iv), which is more or less straightforward if a bit tedious. Note that claims on regular conditional probabilities can be made rigorous, along the lines of the proof of Proposition 11 Let $Z_{1}$ (resp. $Z_{2}$ ) the leftmost (resp. rightmost) point swallowed by an $\operatorname{SLE}_{\left(0, y_{1}, y_{2}\right)}(\kappa, \kappa-4, \kappa-4)$. Then $\left(Z_{1}, Z_{2}\right)$ has density:

$$
-\frac{\partial^{2}}{\partial u_{1} \partial u_{2}} k\left(u_{1}, u_{2}\right) \mathbf{1}_{y_{1} \leq u_{1} \leq 0 \leq u_{2} \leq y_{2}} d u_{1} d u_{2} / k\left(y_{1}, y_{2}\right) .
$$

Conditioning on $\left\{u_{1} \leq Z_{1}, Z_{2} \leq u_{2}\right\}$, we get an $\operatorname{SLE}_{\left(0, u_{1}, u_{2}\right)}(\kappa, \kappa-4, \kappa-4)$. As in the one-sided case, if we define $U_{t}^{i}=g_{t}\left(u_{i}\right)-W_{t}, V_{t}^{i}=g_{t}\left(v_{i}\right)-W_{t}, i=1,2$, it follows that:

$\mathbb{E}^{\uparrow \uparrow}\left(f\left(V_{t}^{1}, V_{t}^{2}\right) \mathbf{1}_{\tau_{v_{1}}>t, \tau_{v_{2}}>t} \mid \begin{array}{l}u_{1} \leq Z_{1} \leq v_{1} \\ v_{2} \leq Z_{2} \leq u_{2}\end{array}\right)=\frac{\mathbb{E}\left(f\left(V_{t}^{1}, V_{t}^{2}\right) \mathbf{1}_{\tau_{v_{1}}>t, \tau_{v_{2}}>t} \tilde{k}\left(U_{t}^{1}, U_{t}^{2}, V_{t}^{1}, V_{t}^{2}\right)\right)}{\tilde{k}\left(u_{1}, u_{2}, v_{1}, v_{2}\right)}$

where $\tilde{k}$ is defined by:

$$
\begin{aligned}
\tilde{k}\left(u_{1}, u_{2}, v_{1}, v_{2}\right) & \stackrel{\text { def }}{=} k\left(u_{1}, u_{2}\right)-k\left(v_{1}, u_{2}\right)-k\left(u_{1}, v_{2}\right)+k\left(v_{1}, v_{2}\right) \\
& =\partial_{u_{1} u_{2}} k\left(u_{1}, u_{2}\right)\left(u_{1}-v_{1}\right)\left(u_{2}-v_{2}\right)+o\left(\left(u_{1}-v_{1}\right)\left(u_{2}-v_{2}\right)\right)
\end{aligned}
$$

so that $\mathbb{P}_{\left(u_{1}, u_{2}\right)}^{\uparrow \uparrow}\left(Z_{1} \leq v_{1}, Z_{2} \geq v_{2}\right)=\tilde{k}\left(u_{1}, u_{2}, v_{1}, v_{2}\right) / k\left(u_{1}, u_{2}\right)$.

Taking the limit as $v_{1} \searrow u_{1}, v_{2} \nearrow u_{2}$, one gets a regular conditional probability for the process stopped at $\tau_{Z_{1}} \wedge \tau_{Z_{2}}$ :

$$
\begin{aligned}
& \mathbb{E}^{\uparrow \uparrow}\left(f\left(U_{t}^{1}, U_{t}^{2}\right) \mathbf{1}_{\tau_{u_{1}}>t, \tau_{u_{2}}>t} \mid Z_{1} \in d u_{1}, Z_{2} \in d u_{2}\right)= \\
& \frac{\mathbb{E}\left(f\left(U_{t}^{1}, U_{t}^{2}\right) \mathbf{1}_{\tau_{Z_{1}>t}, \tau_{Z_{2}>t}}\left(-g_{t}^{\prime}\left(u_{1}\right) g_{t}^{\prime}\left(u_{2}\right) \partial_{u_{1} u_{2}} k\left(U_{t}^{1}, U_{t}^{2}\right)\right)\right)}{-\partial_{u_{1} u_{2}} k\left(u_{1}, u_{2}\right)} d u_{1} d u_{2} .
\end{aligned}
$$

Let $Q \downarrow \downarrow$ be the semigroup of this conditional process killed at $\tau_{Z_{1}} \wedge \tau_{Z_{2}}$. Suppose that $\tau_{Z_{1}}<\tau_{Z_{2}}$; between $\tau_{Z_{1}}$ and $\tau_{Z_{2}}$, the law of the process $\left(Z_{t}^{1}, Z_{t}^{2}\right)=\left(g_{t}\left(Z_{1}^{-}\right)-\right.$ $\left.W_{t}, g_{t}\left(Z_{2}^{+}\right)-W_{t}\right)$ is given by:

$$
\begin{aligned}
\mathbb{E}^{\uparrow \uparrow}\left(f\left(Z_{\tau_{Z_{1}}+t}^{1}, Z_{\tau_{Z_{1}}+t}^{2}\right) \mathbf{1}_{\left.\tau_{Z_{2}>\tau_{Z_{1}}+t} \mid \mathcal{F}_{\tau_{Z_{1}}}, Z_{1} \in d v_{1}, Z_{2} \in d v_{2}\right)=}\right. & \frac{\mathbb{E}\left(f\left(U_{t}^{1}, U_{t}^{2}\right) \mathbf{1}_{\tau_{u_{2}}>t} g_{t}^{\prime}\left(u_{2}\right) \partial_{u_{2}} k\left(U_{t}^{1}, U_{t}^{2}\right)\right)}{\partial_{u_{2}} k\left(u_{1}, u_{2}\right)} d v_{1} d v_{2}
\end{aligned}
$$

where $u_{i}=Z_{\tau_{Z_{1}} \wedge \tau_{Z_{2}}}^{i}, U_{t}^{i}=g_{t}\left(u^{i}\right)-W_{t}, i=1,2$. Symmetrically, if $\tau_{Z_{2}}<\tau_{Z_{1}}$, then the law of the process between $\tau_{Z_{2}}$ and $\tau_{Z_{1}}$ is given by:

$$
\begin{aligned}
\mathbb{E}^{\uparrow \uparrow}\left(f\left(U_{\tau_{Z_{2}}+t}^{1}, U_{\tau_{Z_{2}}+t}^{2}\right)\right. & \left.\mathbf{1}_{\tau_{Z_{1}}>\tau_{Z_{2}}+t} \mid \mathcal{F}_{\tau_{Z_{2}}}, Z_{1} \in d v_{1}, Z_{2} \in d v_{2}\right)= \\
& \frac{\mathbb{E}\left(f\left(U_{t}^{1}, U_{t}^{2}\right) \mathbf{1}_{\tau_{u_{1}}>t} g_{t}^{\prime}\left(u_{1}\right) \partial_{u_{1}} k\left(U_{t}^{1}, U_{t}^{2}\right)\right)}{\partial_{u_{1}} k\left(u_{1}, u_{2}\right)} d v_{1} d v_{2} .
\end{aligned}
$$


We sum up this description of an $\operatorname{SLE}_{\left(0, y_{1}, y_{2}\right)}(\kappa, \kappa-4, \kappa-4)$ conditionally on $\left(Z_{1}, Z_{2}\right)=\left(u_{1}, u_{2}\right)$ (in the regular conditional probability sense). Specifically, we give a path decomposition for $\left(Z^{1}, Z^{2}\right)$, where $Z_{t}^{i}=g_{t}\left(Z_{i}\right)-W_{t} ; f$ is some bounded Borel function on $\mathbb{R}^{-} \times \mathbb{R}^{+}$, and $\tau_{i}=\tau_{Z_{i}}$.

- Up to time $\tau_{1} \wedge \tau_{2},\left(Z_{s}^{1}, Z_{s}^{2}\right)_{s \leq \tau_{1} \wedge \tau_{2}}$ is a Markov process starting from $\left(Z_{1}, Z_{2}\right)$ with semigroup:

$$
Q_{t}^{\downarrow \downarrow} f\left(u_{1}, u_{2}\right)=\frac{\mathbb{E}\left(f\left(U_{t}^{1}, U_{t}^{2}\right) \mathbf{1}_{\tau_{u_{1}}>t, \tau_{u_{2}}>t}\left(-g_{t}^{\prime}\left(u_{1}\right) g_{t}^{\prime}\left(u_{2}\right) \partial_{u_{1} u_{2}} k\left(U_{t}^{1}, U_{t}^{2}\right)\right)\right)}{-\partial_{u_{1} u_{2}} k\left(u_{1}, u_{2}\right)} .
$$

- If $\tau_{1}<\tau_{2}$, then $\left(Z_{s+\tau_{1}}^{1}, Z_{s+\tau_{1}}^{2}\right)_{s \leq \tau_{2}-\tau_{1}}$ is a Markov process starting from $\left(0^{-}, Z_{\tau_{1}}^{2}\right)$ with semigroup:

$$
Q_{t}^{\uparrow \downarrow} f\left(u_{1}, u_{2}\right)=\frac{\mathbb{E}\left(f\left(U_{t}^{1}, U_{t}^{2}\right) \mathbf{1}_{\tau_{u_{1}}>t, \tau_{u_{2}}>t}\left(g_{t}^{\prime}\left(u_{2}\right) \partial_{u_{2}} k\left(U_{t}^{1}, U_{t}^{2}\right)\right)\right)}{\partial_{u_{2}} k\left(u_{1}, u_{2}\right)} .
$$

- If $\tau_{2}<\tau_{1}$, then $\left(Z_{s+\tau_{2}}^{1}, Z_{s+\tau_{2}}^{2}\right)_{s \leq \tau_{1}-\tau_{2}}$ is a Markov process starting from $\left(Z_{\tau_{2}}^{1}, 0^{+}\right)$with semigroup:

$$
Q_{t}^{\downarrow \uparrow} f\left(u_{1}, u_{2}\right)=\frac{\mathbb{E}\left(f\left(U_{t}^{1}, U_{t}^{2}\right) \mathbf{1}_{\tau_{u_{1}}>t, \tau_{u_{2}}>t}\left(-g_{t}^{\prime}\left(u_{1}\right) \partial_{u_{1}} k\left(U_{t}^{1}, U_{t}^{2}\right)\right)\right)}{-\partial_{u_{1}} k\left(u_{1}, u_{2}\right)} .
$$

- After time $\tau=\tau_{1} \vee \tau_{2}$, the process $\left(Z_{\tau+s}^{1}, Z_{\tau+s}^{2}\right)_{s}$ is a Markov process starting from $\left(Z_{\tau}^{1}, Z_{\tau}^{2}\right)$ with semigroup:

$$
Q_{t}^{\uparrow \uparrow} f\left(u_{1}, u_{2}\right)=\frac{\mathbb{E}\left(f\left(U_{t}^{1}, U_{t}^{2}\right) \mathbf{1}_{\tau_{u_{1}}>t, \tau_{u_{2}>t}}\left(k\left(U_{t}^{1}, U_{t}^{2}\right)\right)\right)}{k\left(u_{1}, u_{2}\right)} .
$$

We now consider the following situation: $\left(g_{t}\right)$ (resp. $\left(K_{t}\right)$ ) is the family of conformal equivalences (resp. hulls) associated with an $\operatorname{SLE}_{\left(0, y_{1}, y_{2}\right)}(\kappa, \kappa-4, \kappa-$ 4), $y_{1} \leq 0 \leq y_{2}, W$ is the driving process of this SLE, $Y_{t}^{i}=g_{t}\left(y_{i}\right)-W_{t}$. For any time $t$, let $X_{t}^{1}$ (resp. $X_{t}^{2}$ ) be the leftmost (resp. rightmost) point swallowed by $\left(g_{t}^{-1}\left(K_{t+s} \backslash K_{t}\right)-W_{t}\right)_{s}$, which is an $\operatorname{SLE}_{\left(0, Y_{t}^{1}, Y_{t}^{2}\right)}(\kappa, \kappa-4, \kappa-4)$ process. Alternatively, one may define:

$$
\left(X_{t}^{1}, X_{t}^{2}\right)=\left(g_{t}\left(\gamma_{\sigma_{t}^{1}}\right)-W_{t}, g_{t}\left(\gamma_{\sigma_{t}^{2}}\right)-W_{t}\right)
$$

where $\sigma_{t}^{1}$ (resp. $\sigma_{t}^{2}$ ) is the last time before $t$ spent by the trace of the left (resp. right) boundary of the final hull $K_{\infty}$. Cuttimes can be characterized as zeroes of the joint process $\left(X^{1}, X^{2}\right)$; indeed, if $X^{1}$ and $X^{2}$ vanish at time $t$, then by definition $\gamma_{t}$ lies on the left and right boundaries of $K_{\infty}$, in other words $\gamma_{t}$ is a cutpoint of $K_{\infty}$.

We have seen that $\left(X^{1}, X^{2}\right)$ is a continuous process, and that for $t>0$, the law of $\left(X_{t}^{1}, X_{t}^{2}\right)$ conditionally on $\mathcal{Y}_{t}=\sigma\left(\left(Y_{s}^{1}, Y_{s}^{2}\right)_{0 \leq s \leq t}\right)$ is $\Upsilon\left(\left(Y_{t}^{1}, Y_{t}^{2}\right)\right.$,. $)$, where $\Upsilon$ is the transition kernel $\mathbb{R}^{-} \times \mathbb{R}^{+} \rightarrow \mathbb{R}^{-} \times \mathbb{R}^{+}$specified by:

$$
(\Upsilon f)\left(y_{1}, y_{2}\right)=\int_{y_{1}}^{0} \int_{0}^{y_{2}} f\left(x_{1}, x_{2}\right) \frac{-\partial_{x_{1} x_{2}} k\left(x_{1}, x_{2}\right)}{k\left(y_{1}, y_{2}\right)} d x_{1} d x_{2}
$$


where $f$ is some bounded Borel function on $\mathbb{R}^{-} \times \mathbb{R}^{+}$. We will also need onesided versions of this intertwining kernel. More precisely, the conditional law of $\left(X_{t}^{1}, X_{t}^{2}\right)$ given $\left(X_{t}^{1}, Y_{t}^{2}\right)$ is $\Upsilon^{1}\left(\left(X_{t}^{1}, Y_{t}^{2}\right),.\right)$, where:

$$
\left(\Upsilon^{1} f\right)\left(x_{1}, y_{2}\right)=\int_{0}^{y_{2}} f\left(x_{1}, x_{2}\right) \frac{-\partial_{x_{1} x_{2}} k\left(x_{1}, x_{2}\right)}{-\partial_{x_{1}} k\left(x_{1}, y_{2}\right)} d x_{2} .
$$

Symmetrically, the law of $\left(X_{t}^{1}, X_{t}^{2}\right)$ given $\left(Y_{t}^{1}, X_{t}^{2}\right)$ is $\Upsilon^{2}\left(\left(Y_{t}^{1}, X_{t}^{2}\right),.\right)$, where:

$$
\left(\Upsilon^{2} f\right)\left(y_{1}, x_{2}\right)=\int_{y_{1}}^{0} f\left(x_{1}, x_{2}\right) \frac{-\partial_{x_{1} x_{2}} k\left(x_{1}, x_{2}\right)}{\partial_{x_{2}} k\left(y_{1}, x_{2}\right)} d x_{1} .
$$

We will prove that $\left(X^{1}, X^{2}\right)$ is a Markov process; we now describe its semigroup. We have seen that the law of $\left(X^{1}, X^{2}\right)$ starting from $x_{1}<0<x_{2}$ and killed at $\tau_{X_{1}} \wedge \tau_{X_{2}}$ is that of a Markov process with (non conservative) semigroup $Q^{\downarrow \downarrow}$. So the problem is to extend trajectories of $\left(X^{1}, X^{2}\right)$ after $\tau_{X_{1}} \wedge \tau_{X_{2}}$; for this, we will need the semigroups $Q^{\uparrow \downarrow}, Q^{\downarrow \uparrow}, Q^{\uparrow \uparrow}$, and the intertwining kernels $\Upsilon^{2}, \Upsilon^{1}, \Upsilon$.

Let $\left(X_{1}^{\downarrow \downarrow}, X_{2}^{\downarrow \downarrow}\right)$ be a Markov process with (non conservative) semigroup $Q^{\downarrow \downarrow}$, started from $\left(x_{1}, x_{2}\right), x_{1} \leq 0 \leq x_{2}$, and $T$ be its lifetime; then either $X_{1, T}^{\downarrow \downarrow}$ or $X_{2, T}^{\downarrow \downarrow}$ vanish. If $X_{1, T}^{\downarrow \downarrow}=0$ (resp. $X_{2, T}^{\downarrow \downarrow}=0$ ), consider an independent Markov pro$\operatorname{cess}\left(Y_{1}^{\uparrow \downarrow}, X_{2}^{\uparrow \downarrow}\right)\left(\right.$ resp. $\left.\left(X_{1}^{\downarrow \uparrow}, Y_{2}^{\downarrow \uparrow}\right)\right)$ with semigroup $Q^{\uparrow \downarrow}\left(\right.$ resp. $\left.Q^{\downarrow \uparrow}\right)$ started from $\left(0^{-}, X_{2, T}^{\downarrow \downarrow}\right)$ (resp. $\left.\left(X_{1, T}^{\downarrow \downarrow}, 0^{+}\right)\right)$; let $T^{\prime}$ be its lifetime. Finally, let $\left(Y_{1}^{\uparrow \uparrow}, Y_{2}^{\uparrow \uparrow}\right)$ be an independent Markov process with semigroup $Q^{\uparrow \uparrow}$ started from $\left(Y_{1}^{\uparrow \downarrow}, X_{2}^{\uparrow \downarrow}\right)_{T^{\prime}}$ (resp. $\left(X_{1}^{\downarrow \uparrow}, Y_{2}^{\downarrow \uparrow}\right)_{T^{\prime}}$ ). These processes are defined on some probability space; the notations $\mathbf{P}, \mathbf{E}$ refer to this space. One can now define:

$$
\begin{aligned}
\left(\tilde{Q}_{t}^{\downarrow \downarrow} f\right)\left(x_{1}, x_{2}\right)= & \mathbf{E}\left(\mathbf{1}_{t \leq T} f\left(X_{1, t}^{\downarrow \downarrow}, X_{2, t}^{\downarrow \downarrow}\right)\right) \\
& +\mathbf{E}\left(\mathbf{1}_{T<t \leq T+T^{\prime}} \mathbf{1}_{X_{1, T}^{\downarrow \downarrow}=0}\left(\Upsilon^{2} f\right)\left(Y_{1, t-T}^{\uparrow \downarrow}, X_{2, t-T}^{\uparrow \downarrow}\right)\right) \\
& +\mathbf{E}\left(\mathbf{1}_{T<t \leq T+T^{\prime}} \mathbf{1}_{X_{2, T}^{\downarrow \downarrow}=0}\left(\Upsilon^{1} f\right)\left(X_{1, t-T}^{\downarrow \uparrow}, Y_{2, t-T}^{\downarrow \uparrow}\right)\right) \\
& +\mathbf{E}\left(\mathbf{1}_{T+T^{\prime}<t}(\Upsilon f)\left(Y_{1, t-T-T^{\prime}}^{\uparrow \uparrow}, Y_{2, t-T-T^{\prime}}^{\uparrow \uparrow}\right)\right) .
\end{aligned}
$$

It will also be convenient to extend the non conservative semigroups $Q^{\uparrow \downarrow}$, $Q^{\downarrow \uparrow}$. Denote by $\iota$ the following involution of $\mathbb{R}^{-} \times \mathbb{R}^{+}: \iota\left(\left(u_{1}, u_{2}\right)\right)=\left(-u_{2},-u_{1}\right)$. Then $Q^{\uparrow \downarrow}=\iota Q^{\downarrow \uparrow} \iota, \Upsilon^{1}=\iota \Upsilon^{2} \iota$, so that we can restrict to $Q^{\uparrow \downarrow}$ for instance. As above, we consider a Markov process $\left(Y_{1}^{\uparrow \downarrow}, X_{2}^{\uparrow \downarrow}\right)$ with semigroup $Q^{\uparrow \downarrow}$ started from $\left(y_{1} \leq 0 \leq x_{2}\right)$; let $T$ be its lifetime (i.e. $X_{2}^{\uparrow \downarrow}$ is positive before $T$ and vanishes at time $T)$. Let $\left(Y_{1}^{\uparrow \uparrow}, Y_{2}^{\uparrow \uparrow}\right)$ be a Markov process with semigroup $Q^{\uparrow \uparrow}$ independent of the former conditionally on its starting state $\left(Y_{1, T}^{\uparrow \downarrow}, 0^{+}\right)$. We will 
also need another two Markov kernels (from $\mathbb{R}^{-} \times \mathbb{R}^{+}$to itself):

$$
\begin{aligned}
& \left(\Upsilon_{1} f\right)\left(y_{1}, y_{2}\right)=\int_{0}^{y_{2}} f\left(y_{1}, x_{2}\right) \frac{\partial_{x_{2}} k\left(y_{1}, x_{2}\right)}{k\left(y_{1}, y_{2}\right)} d x_{2} \\
& \left(\Upsilon_{2} f\right)\left(y_{1}, y_{2}\right)=\int_{y_{1}}^{0} f\left(x_{1}, y_{2}\right) \frac{-\partial_{x_{1}} k\left(x_{1}, y_{2}\right)}{k\left(y_{1}, y_{2}\right)} d x_{1}
\end{aligned}
$$

so that the conditional law of $\left(Y_{t}^{1}, X_{t}^{2}\right)$ given $\left(Y_{t}^{1}, Y_{t}^{2}\right)$ is $\Upsilon_{1}\left(\left(Y_{t}^{1}, Y_{t}^{2}\right),.\right)$, and the conditional law of $\left(X_{t}^{1}, Y_{t}^{2}\right)$ given $\left(Y_{t}^{1}, Y_{t}^{2}\right)$ is $\Upsilon_{2}\left(\left(Y_{t}^{1}, Y_{t}^{2}\right),.\right)$; the identities $\Upsilon_{1} \Upsilon^{2}=\Upsilon_{2} \Upsilon^{1}=\Upsilon$ are then obvious. We now define:

$$
\left(\tilde{Q}_{t}^{\uparrow \downarrow} f\right)\left(y_{1}, x_{2}\right)=\mathbf{E}\left(\mathbf{1}_{t \leq T} f\left(Y_{1, t}^{\uparrow \downarrow}, X_{2, t}^{\uparrow \downarrow}\right)\right)+\mathbf{E}\left(\mathbf{1}_{T<t}\left(\Upsilon_{1} f\right)\left(Y_{1, t-T}^{\uparrow \uparrow}, Y_{2, t-T}^{\uparrow \uparrow}\right)\right) .
$$

Note that the semigroup properties $\tilde{Q}_{t+s}^{\downarrow \downarrow}=\tilde{Q}_{t}^{\downarrow \downarrow} \tilde{Q}_{s}^{\downarrow \downarrow}, \tilde{Q}_{t+s}^{\uparrow \downarrow}=\tilde{Q}_{t}^{\uparrow \downarrow} \tilde{Q}_{s}^{\uparrow \downarrow}$, are not obvious for the moment. Let $\mathcal{X}_{t}^{i}=\sigma\left(\left(X_{s}^{i}\right)_{0 \leq s \leq t}\right), \mathcal{X}=\mathcal{X}^{1} \vee \mathcal{X}^{2}$; we can finally state:

Proposition 9. The process $\left(X^{1}, X^{2}\right)$ (resp. $\left(Y^{1}, X^{2}\right),\left(X^{1}, Y^{2}\right)$ ) is Markov with semigroup $\tilde{Q}^{\downarrow \downarrow}$ (resp. $\tilde{Q}^{\uparrow \downarrow}, \tilde{Q}^{\downarrow \uparrow}$ ) in the filtration $\mathcal{X} \vee \mathcal{Y}$ (resp. $\mathcal{X}^{2} \vee \mathcal{Y}$, $\left.\mathcal{X}^{1} \vee \mathcal{Y}\right)$. Moreover, the following intertwining relations hold:

$$
\begin{array}{llrl}
Q^{\uparrow \uparrow} \Upsilon_{1} & =\Upsilon_{1} \tilde{Q}^{\uparrow \downarrow} & & Q^{\uparrow \uparrow} \Upsilon=\Upsilon \tilde{Q}^{\downarrow \downarrow} \\
Q^{\uparrow \uparrow} \Upsilon_{2}=\Upsilon_{2} \tilde{Q}^{\downarrow \uparrow} & & \tilde{Q}^{\uparrow \downarrow} \Upsilon^{2}=\Upsilon^{2} \tilde{Q}^{\downarrow \downarrow} \\
& & \tilde{Q}^{\downarrow \uparrow} \Upsilon^{1}=\Upsilon^{1} \tilde{Q}^{\downarrow \downarrow}
\end{array}
$$

Proof. First, we consider the statement for $\left(Y^{1}, X^{2}\right)$ (the case of $\left(X^{1}, Y^{2}\right)$ being symmetric). Let $0 \leq s \leq t$. As in the one-sided case, we see that $\left(Y_{s+u}^{1}, X_{s+u}^{2}\right)_{u}$ is independent from $\mathcal{X}_{s}^{2} \vee \mathcal{Y}_{s}$ conditionally on $\left(Y_{s}^{1}, X_{s}^{2}\right)$; this follows from the Markov property of $\operatorname{SLE}(\kappa, \kappa-4, \kappa-4)$, Proposition 2 (ii), and the fact that $\mathcal{X}_{s}^{i} \vee \mathcal{Y}_{s}=\sigma\left(\mathcal{Y}_{s}, X_{s}^{i}\right), i=1,2$. Moreover, $\left(Y_{s+u}^{1}, X_{s+u}^{2}\right)_{u}$ killed at $\tau_{2}$ is Markov with semigroup $Q^{\uparrow \downarrow}$, where $\tau_{2}$ is the first time after $s$ at which $X^{2}$ vanishes.

Let $\tau$ be an $\mathcal{X}^{2} \vee \mathcal{Y}$ stopping time such that $X_{\tau}^{2}=0$ a.s. . Then

$$
\left(\tilde{Y}^{1}, \tilde{Y}^{2}\right)_{u}=\left(Y_{\tau+u}^{1}, g_{\tau+u}\left(g_{\tau}^{-1}\left(W_{\tau}^{+}\right)\right)-W_{\tau+u}\right)_{u}
$$

is a Markov process with semigroup $Q^{\uparrow \uparrow}$, independent from $\left(\mathcal{X}^{2} \vee \mathcal{Y}\right)_{\tau}$ conditionally on its starting state $\left(Y_{\tau}^{1}, 0^{+}\right)$. As in Proposition 3 this follows from the decomposition of an $\operatorname{SLE}(\kappa, \kappa-4, \kappa-4)$ conditionally on its rightmost swallowed point. The process $\left(\tilde{Y}^{1}, \tilde{Y}^{2}\right)_{u}$ is but the driving mechanism of an $\operatorname{SLE}(\kappa, \kappa-4, \kappa-4)$ independent from $\mathcal{X}_{t}^{2} \vee \mathcal{Y}_{t}$ conditionally on $Y_{t}^{1}$; let $\left(\tilde{X}^{1}, \tilde{X}^{2}\right)$ be the associated process (i.e. $\tilde{X}_{u}^{2}$ is the rightmost swallowed point after time $u)$. Then $\tilde{X}_{u}^{2}=X_{\tau+u}^{2}$ and the conditional law of $\left(\tilde{Y}_{u}^{1}, \tilde{X}_{u}^{2}\right)$ given $\left(\tilde{Y}_{u}^{1}, \tilde{Y}_{u}^{2}\right)$ is $\Upsilon_{1}\left(\left(\tilde{Y}_{u}^{1}, \tilde{Y}_{u}^{2}\right),.\right)$. Setting $\tau=\tau_{2}$, one gets:

$$
\begin{aligned}
\mathbb{E}\left(f\left(Y_{t}^{1}, X_{t}^{2}\right) \mid \mathcal{X}_{s}^{2}, \mathcal{Y}_{s}\right) & =\left(Q_{t-s}^{\uparrow \downarrow} f\right)\left(Y_{s}^{1}, X_{s}^{2}\right)+\mathbb{E}\left(\mathbf{1}_{\tau_{2} \leq t}\left(Q_{t-\tau_{2}}^{\uparrow \uparrow} \Upsilon_{1} f\right)\left(Y_{\tau_{2}}^{1}, 0^{+}\right)\right) \\
& =\left(\tilde{Q}_{t-s}^{\uparrow \downarrow} f\right)\left(Y_{s}^{1}, X_{s}^{2}\right) .
\end{aligned}
$$


Hence it appears that $\left(Y^{1}, X^{2}\right)$ is an homogeneous $\mathcal{X}^{2} \vee \mathcal{Y}$-Markov process with semigroup $\tilde{Q}_{t-s}^{\uparrow \downarrow}$. As for the intertwining relation, we get as before:

$$
\begin{aligned}
\mathbb{E}\left(f\left(Y_{t}^{1}, X_{t}^{2}\right) \mid \mathcal{Y}_{s}\right) & =\mathbb{E}\left(f\left(Y_{t}^{1}, X_{t}^{2}\right)\left|\mathcal{Y}_{t}\right| \mathcal{Y}_{s}\right)=\mathbb{E}\left(\left(\Upsilon_{1} f\right)\left(Y_{t}^{1}, Y_{t}^{2}\right) \mid \mathcal{Y}_{s}\right)=\left(Q_{t-s}^{\uparrow \uparrow} \Upsilon_{1} f\right)\left(Y_{s}^{1}, Y_{s}^{2}\right) \\
& =\mathbb{E}\left(f\left(Y_{t}^{1}, X_{t}^{2}\right)\left|\mathcal{Y}_{s}, \mathcal{X}_{s}^{2}\right| \mathcal{Y}_{s}\right)=\mathbb{E}\left(\left(\tilde{Q}_{t-s}^{\uparrow \downarrow} f\right)\left(Y_{s}^{1}, X_{s}^{2}\right) \mid \mathcal{Y}_{s}\right)=\left(\Upsilon_{1} \tilde{Q}_{t-s}^{\uparrow \downarrow} f\right)\left(Y_{s}^{1}, Y_{s}^{2}\right) .
\end{aligned}
$$

We now turn to $\left(X^{1}, X^{2}\right)$, building on the previous result. Let $0 \leq s \leq t$; we have seen that $\left(X_{s+u}^{1}, X_{s+u}^{2}\right)_{u}$ is independent from $\mathcal{X}_{s} \vee \mathcal{Y}_{s}$ conditionally on $\left(X_{s}^{1}, X_{s}^{2}\right)$ (recall that $\left.\mathcal{X}_{s} \vee \mathcal{Y}_{s}=\sigma\left(\mathcal{Y}_{s}, X_{s}^{1}, X_{s}^{2}\right)\right)$. Moreover, $\left(X_{s+u}^{1}, X_{s+u}^{2}\right)_{u}$ killed at $\tau_{1} \wedge \tau_{2}$ is Markov with semigroup $Q^{\downarrow \downarrow}$, where $\tau_{i}$ is the first time after $s$ at which $X^{i}$ vanishes.

Suppose that $\tau_{1}<\tau_{2}$; then $\left(\tilde{Y}_{u}^{1}, \tilde{X}_{u}^{2}\right)_{u}=\left(g_{\tau_{1}+u}\left(g_{\tau_{1}}^{-1}\left(W_{\tau_{1}+u}^{-}\right)\right)-W_{\tau_{1}+u}, X_{\tau_{1}+u}^{2}\right)_{u}$, killed at $\tau_{2}-\tau_{1}$, is a Markov process with semigroup $\tilde{Q}^{\uparrow \downarrow}$, independent from $(\mathcal{X} \vee \mathcal{Y})_{\tau_{1}}$ conditionally on its starting state $\left(0^{-}, X_{\tau_{1}}^{2}\right)$. Moreover, the law of $\left(X_{\tau_{1}+u}^{1}, X_{\tau_{1}+u}^{2}\right)$ given $\left(\tilde{Y}_{u}^{1}, \tilde{X}_{u}^{2}\right)$ is $\Upsilon^{2}\left(\left(\tilde{Y}_{u}^{1}, \tilde{X}_{u}^{2}\right),.\right)$. It follows that:

$$
\begin{aligned}
\mathbb{E}\left(f\left(X_{t}^{1}, X_{t}^{2}\right) \mid \mathcal{X}_{s}, \mathcal{Y}_{s}\right)= & \left(Q^{\downarrow \downarrow} f\right)\left(X_{s}^{1}, X_{s}^{2}\right)+\mathbb{E}\left(\mathbf{1}_{\tau_{1} \leq t \wedge \tau_{2}}\left(\tilde{Q}_{t-\tau_{1}}^{\uparrow \downarrow} \Upsilon^{2} f\right)\left(0^{-}, X_{\tau_{1}}^{2}\right)\right) \\
& +\mathbb{E}\left(\mathbf{1}_{\tau_{2} \leq t \wedge \tau_{1}}\left(\tilde{Q}_{t-\tau_{2}}^{\downarrow \uparrow} \Upsilon^{1} f\right)\left(X_{\tau_{2}}^{1}, 0^{+}\right)\right) \\
= & \left(\tilde{Q}_{t-s}^{\downarrow \downarrow} f\right)\left(X_{s}^{1}, X_{s}^{2}\right),
\end{aligned}
$$

taking into account the previous decomposition of $\tilde{Q}^{\uparrow \downarrow}, \tilde{Q}^{\downarrow \uparrow}$. The intertwining relations follow as before. Note that $Q^{\uparrow \uparrow} \Upsilon_{1}=\Upsilon_{1} \tilde{Q}^{\uparrow \downarrow}, \tilde{Q}^{\uparrow \downarrow} \Upsilon^{2}=\Upsilon^{2} \tilde{Q}^{\downarrow \downarrow}$, and $\Upsilon=\Upsilon_{1} \Upsilon^{2}$ imply that $Q^{\uparrow \uparrow} \Upsilon=\Upsilon \tilde{Q}^{\downarrow \downarrow}$.

From here, as we can no longer rely on classical results for Bessel processes, we have to prove the existence of a local time at $(0,0)$ for the Markov process $\left(X^{1}, X^{2}\right)$, whose right-continuous inverse is a stable process. The key tool is a local martingale, acting in many respects as a "scale function" for $\left(X^{1}, X^{2}\right)$.

Lemma 10. Let $4<\kappa<8$. If $\left(X_{1}, X_{2}\right)$ is a Markov process with semigroup $\tilde{Q}^{\downarrow \downarrow}$ started from $\left(x_{1}, x_{2}\right), x_{1} \leq 0 \leq x_{2}$, then the process:

$$
R_{t} \stackrel{\text { def }}{=} \frac{\left(X_{2, t}-X_{1, t}\right)^{4-\kappa / 2}}{1+(4-\kappa / 2)\left(X_{1, t} X_{2, t}\right) /\left(X_{2, t}-X_{1, t}\right)^{2}} \mathbf{1}_{t \leq T},
$$

where $T=\inf \left(t>0: X_{1, t}=X_{2, t}=0\right)$, is an $\mathcal{X} \vee \mathcal{Y}$ - local martingale.

Proof. Note that it is not obvious that $R$ is a semimartingale. It is easily seen that under $\mathbb{P}$, if $u_{1}<0<u_{2}$, the following semimartingale:

$A_{t}\left(u_{1}, u_{2}\right) \stackrel{\text { def }}{=} g_{t}^{\prime}\left(u_{1}\right) g_{t}^{\prime}\left(u_{2}\right)\left(W_{t}-g_{t}\left(u_{1}\right)\right)^{d-1}\left(g_{t}\left(u_{2}\right)-W_{t}\right)^{d-1}\left(g_{t}\left(u_{2}\right)-g_{t}\left(u_{1}\right)\right)^{(\kappa / 2)(d-1)^{2}}$

is a local martingale (as long as $u_{1}$ and $u_{2}$ are not swallowed). Suppose that $x_{1}<0<x_{2}$. If $\tau_{i}=\inf \left(t>0: X_{i, t}=0\right)$, then $\left(X_{1}, X_{2}\right)$ killed at $\tau=\tau_{1} \wedge \tau_{2}$ 
is Markov with semigroup $Q^{\downarrow \downarrow}$. Let $M_{n}=\inf \left(t>0:\left(-X_{1, t}\right) \vee X_{2, t} \geq n\right)$ be a sequence of stopping times. Then:

$$
\begin{aligned}
\mathbb{E}^{\downarrow \downarrow}\left(R_{t \wedge M_{n} \wedge \tau} \mid \mathcal{X}_{0} \vee \mathcal{Y}_{0}\right) / R_{0} & =\frac{\mathbb{E}\left(R_{t \wedge M_{n} \wedge \tau}\left(g_{t}^{\prime}\left(x_{1}\right) g_{t}^{\prime}\left(x_{2}\right) \partial_{u_{1} u_{2}} k\left(X_{1, t}, X_{2, t}\right)\right)\right)}{R_{0} \partial_{u_{1} u_{2}} k\left(x_{1}, x_{2}\right)} \\
& =\frac{\mathbb{E}\left(A_{t \wedge M_{n} \wedge \tau}\left(x_{1}, x_{2}\right)\right)}{A_{0}\left(x_{1}, x_{2}\right)}=1
\end{aligned}
$$

which is enough to prove that $\left(R_{t}^{\tau}\right)$ is a local martingale, given the Markov property of $X$. Note that we have used the identity:

$$
\partial_{u_{1} u_{2}} k\left(u_{1}, u_{2}\right)=d^{2}(\kappa / 2-1)\left(\frac{1}{u_{1} u_{2}}+\frac{4-\kappa / 2}{\left(u_{2}-u_{1}\right)^{2}}\right) k\left(u_{1}, u_{2}\right) .
$$

We now consider the case $x_{1}=0<x_{2}$ (the case $x_{1}<0=x_{2}$ being symmetrical). Suppose that $y_{1}<0<x_{2}$. Then $\left(Y_{1}, X_{2}\right)$ killed at $\tau_{2}$ is Markov with semigroup $Q^{\uparrow \downarrow}$, and is intertwined with $\left(X_{1}, X_{2}\right)$ via the Markov kernel $\Upsilon^{2}$. Let $\tilde{M}_{n}=$ $\inf \left(t>0:\left(-Y_{1, t}\right) \vee X_{2, t} \geq n\right)$. From the definitions of $Q^{\uparrow \downarrow}$ and $\Upsilon^{2}$, we get:

$$
\mathbb{E}^{\downarrow \downarrow}\left(R_{t \wedge \tilde{M}_{n} \wedge \tau_{2}} \mid \mathcal{X}_{0}^{2} \vee \mathcal{Y}_{0}\right)=\frac{\mathbb{E}\left(\left(\Upsilon^{2} R\right)_{t \wedge \tilde{M}_{n} \wedge \tau_{2}}\left(g_{t}^{\prime}\left(x_{2}\right) \partial_{u_{2}} k\left(Y_{1, t}, X_{2, t}\right)\right)\right)}{\partial_{u_{2}} k\left(y_{1}, x_{2}\right)}
$$

where we define:

$$
\left(\Upsilon^{2} R\right)_{t}=\int_{Y_{1, t}}^{0} r\left(x_{1}, X_{2, t}\right) \frac{-\partial_{u_{1} u_{2}} k\left(x_{1}, X_{2, t}\right)}{\partial_{u_{2}} k\left(Y_{1, t}, X_{2, t}\right)} d x_{1} .
$$

with $r\left(x_{1}, x_{2}\right)=\left(x_{2}-x_{1}\right)^{4-\kappa / 2} /\left(1+(4-\kappa / 2) x_{1} x_{2} /\left(x_{2}-x_{1}\right)^{2}\right)$. It follows that:

$$
\left(\Upsilon^{2} R\right)_{t}\left(g_{t}^{\prime}\left(x_{2}\right) \partial_{u_{2}} k\left(Y_{1, t}, X_{2, t}\right)\right)=\int_{Y_{1, t}}^{0} r\left(x_{1}, X_{2, t}\right)\left(-\partial_{u_{1} u_{2}} k\left(x_{1}, X_{2, t}\right)\right) g_{t}^{\prime}\left(x_{2}\right) d x_{1} \text {. }
$$

This is a local martingale; with the change of variable $x_{1}=g_{t}\left(\tilde{x}_{1}\right)-W_{t}$, one can see it as an integrated version of $A_{t}\left(., x_{2}\right)$. More precisely, one has to check that:

$$
\left(\mathcal{L}-\frac{2}{y_{2}^{2}}\right)\left(\int_{y_{1}}^{0}\left(-x_{1} y_{2}\right)^{-4 / \kappa}\left(y_{2}-x_{1}\right)^{8 / \kappa} d x_{1}\right)=0
$$

where $\mathcal{L}$ is the differential operator $\left(\kappa / 2\left(\partial_{y_{1}}+\partial_{y_{2}}\right)^{2}+2 / y_{1} \partial_{y_{1}}+2 / y_{2} \partial_{y_{2}}\right)$. A simple way to see this is to observe that

$$
\begin{aligned}
\partial_{y_{1}}\left(\mathcal{L}-\frac{2}{y_{2}^{2}}\right)(\ldots) & =\left(\mathcal{L}-\frac{2}{y_{1}^{2}}-\frac{2}{y_{2}^{2}}\right) \partial_{y_{1}}\left(\int_{y_{1}}^{0}\left(-x_{1} y_{2}\right)^{-4 / \kappa}\left(y_{2}-x_{1}\right)^{8 / \kappa} d x_{1}\right) \\
& =-\left(\mathcal{L}-\frac{2}{y_{1}^{2}}-\frac{2}{y_{2}^{2}}\right)\left(\left(-y_{1} y_{2}\right)^{-4 / \kappa}\left(y_{2}-y_{1}\right)^{8 / \kappa}\right)=0
\end{aligned}
$$

and, as $y_{1} \nearrow 0$,

$$
\begin{aligned}
\left(\mathcal{L}-\frac{2}{y_{2}^{2}}\right)(\ldots) & =-\left(\frac{\kappa}{2} \partial_{y_{1}}+\kappa \partial_{y_{2}}+\frac{2}{y_{1}}\right)\left(\left(-y_{1} y_{2}\right)^{-4 / \kappa}\left(y_{2}-y_{1}\right)^{8 / \kappa}\right)+o\left(y_{1}\right) \\
& =-\left(-y_{1} y_{2}\right)^{-4 / \kappa}\left(y_{2}-y_{1}\right)^{8 / \kappa}\left(-\frac{2}{y_{1}}-\frac{4}{y_{2}}+\frac{2}{y_{1}}+\frac{4}{y_{2}-y_{1}}\right)+o\left(y_{1}\right)=o\left(y_{1}\right) .
\end{aligned}
$$


Given that the process $\left(\left(\Upsilon^{2} R\right)_{t}\left(g_{t}^{\prime}\left(x_{2}\right) \partial_{u_{2}} k\left(Y_{1, t}, X_{2, t}\right)\right)\right)$ is a local martingale, we get:

$$
\mathbb{E}^{\downarrow \downarrow}\left(R_{t \wedge \tilde{M}_{n} \wedge \tau_{2}} \mid \mathcal{X}_{0}^{2} \vee \mathcal{Y}_{0}\right)=\mathbb{E}^{\downarrow \downarrow}\left(R_{0} \mid \mathcal{X}_{0}^{2} \vee \mathcal{Y}_{0}\right)=\left(\Upsilon^{2} R\right)_{0}\left(y_{1}, x_{2}\right)
$$

Letting $y_{1} \nearrow 0$, this settles the case $x_{1}=0<x_{2}$. From the Markov property of $\left(X^{1}, X^{2}\right)$, we can conclude that $\left(R_{t}\right)$ is a local martingale.

Finally, we briefly justify the fact that $A_{t}\left(u_{1}, u_{2}\right)$ is a.s. continuous. Suppose e.g. that $\tau_{u_{2}}<\tau_{u_{1}}$; we need to prove that $A_{t}$ goes to 0 as $t \nearrow \tau_{u_{2}}$. Since $4<$ $\kappa<8$, there a.s. exists $v_{2}>u_{2}$ with $\tau_{u_{2}}=\tau_{v_{2}}$. We know that $g_{t}^{\prime}\left(u_{2}\right)\left(v_{2}-u_{2}\right) \leq$ $g_{t}\left(v_{2}\right)-g_{t}\left(u_{2}\right)$. Moreover, from harmonic measure considerations, it appears that $\left(g_{t}\left(v_{2}\right)-W_{t}\right)$ goes to 0 , while the ratio $\left(g_{t}\left(v_{2}\right)-W_{t}\right) /\left(g_{t}\left(u_{2}\right)-W_{t}\right)$ stays bounded, which is enough to conclude.

Before proceeding with the study of "excursions away from cutpoints", we discuss the limiting case $\kappa=8$ (note that for $\operatorname{SLE}(8)$, the set of cut-times has a.s. zero Hausdorff dimension, see [1]).

Lemma 11. For $\kappa=8$, the process:

$$
R_{t} \stackrel{\text { def }}{=}\left(\log \left(X_{2, t}-X_{1, t}\right)-\frac{X_{1, t} X_{2, t}}{\left(X_{2, t}-X_{1, t}\right)^{2}}\right) \mathbf{1}_{t \leq T},
$$

where $T=\inf \left(t>0: X_{1, t}=X_{2, t}=0\right)$, is an $\mathcal{X} \vee \mathcal{Y}$ - local martingale. As a consequence, $(0,0)$ is a polar point for the Markov process $\left(X_{1}, X_{2}\right)$, and the final hull of $\operatorname{SLE}(8,4,4)$ has a.s. no cutpoints.

Proof. In the previous lemma, we considered the local martingales (for $4<\kappa<$ 8):

$$
\begin{aligned}
R_{t}^{(\kappa)} & =\frac{\left(X_{2, t}-X_{1, t}\right)^{4-\kappa / 2}}{1+(4-\kappa / 2)\left(X_{1, t} X_{2, t}\right) /\left(X_{2, t}-X_{1, t}\right)^{2}} \mathbf{1}_{t \leq T} \\
& =\left(1+(4-\kappa / 2)\left(\log \left(X_{2, t}-X_{1, t}\right)-\frac{X_{1, t} X_{2, t}}{\left(X_{2, t}-X_{1, t}\right)^{2}}\right)\right) \mathbf{1}_{t \leq T}+o(\kappa-8),
\end{aligned}
$$

which justifies the definition of $R$ here. It can be checked directly (or using a limiting argument) that for $u_{1}<0<u_{2}$, the semimartingales:

$$
A_{t}\left(u_{1}, u_{2}\right)=g_{t}^{\prime}\left(u_{1}\right) g_{t}^{\prime}\left(u_{2}\right)\left(\log \left(U_{1, t}-U_{2, t}\right)-\frac{U_{1, t} U_{2, t}}{\left(U_{2, t}-U_{1, t}\right)^{2}}\right) \frac{U_{2, t}-U_{1, t}}{\sqrt{-U_{1, t} U_{2, t}}}
$$

where $U_{i, t}=g_{t}\left(u_{i}\right)-W_{t}$, are local martingales under $\mathbb{P}$ (as long as $u_{1}, u_{2}$ are not swallowed). Arguing as in the previous lemma, we get that $R$ is a local martingale. The fact that $(0,0)$ is polar for $\left(X_{1}, X_{2}\right)$ follows immediately. Since cut-times of $\operatorname{SLE}(8,4,4)$ correspond by construction to the zero set of $\left(X_{1}, X_{2}\right)$, one gets that $\operatorname{SLE}(8,4,4)$ as a.s. no cutpoint. 
It is easily seen that if $\operatorname{SLE}(8)$ stopped at a finite time had cutpoints with positive probability, then the final hull of $\operatorname{SLE}(8,4,4)$ would also have cutpoints with positive probability. Consequently, SLE(8) stopped at a finite time has a.s. no cutpoints. One can prove this directly, building on the reversibility of the $\operatorname{SLE}(8)$ trace $(1])$.

We turn back to the case $4<\kappa<8$. Using the above homogeneous "scale function", one can define a local time at $(0,0)$ for $\left(X_{1}, X_{2}\right)$, and compute the index of its (stable) inverse, using Lévy's upcrossings construction. Specifically, consider the level lines:

$$
M_{h}=\left\{\left(x_{1}, x_{2}\right): x_{1} \leq 0 \leq x_{2}, r\left(x_{1}, x_{2}\right)=h\right\}
$$

where $r\left(x_{1}, x_{2}\right)=\left(x_{2}-x_{1}\right)^{4-\kappa / 2} /\left(1+(4-\kappa / 2) x_{1} x_{2} /\left(x_{2}-x_{1}\right)^{2}\right)$. Note that, for $\kappa \in(4,8)$ :

$$
\left(x_{2}-x_{1}\right)^{4-\kappa / 2} \leq r\left(x_{1}, x_{2}\right) \leq \frac{8}{\kappa}\left(x_{2}-x_{1}\right)^{4-\kappa / 2} .
$$

Suppose that the process $\left(X_{1}, X_{2}\right)$ starts from 0 , and define recursively for $h>0$, $n \geq 0$ :

$$
\begin{aligned}
& S_{n}^{h}=\inf \left(t \geq T_{n-1}^{h}: X_{1, t}=X_{2, t}=0\right) \\
& T_{n}^{h}=\inf \left(t \geq S_{n}^{h}:\left(X_{1, t}, X_{2, t}\right) \in M_{h}\right)
\end{aligned}
$$

with the convention $T_{-1}^{h}=0$. The number of upcrossings from level 0 to level $h$ at time $t$ is:

$$
U(h, t)=\sup \left(n \in \mathbb{N}: T_{n-1}^{h} \leq t\right) .
$$

Lemma 12. The a.s. limit $\lim _{n} 2^{-n} U\left(2^{-n}, t\right)$ exists and defines a local time $\left(\ell_{t}\right)$ for the Markov process $\left(X_{1}, X_{2}\right)$ at $(0,0)$. The right-continuous inverse of $\ell$ is a stable subordinator with index $(2-\kappa / 4)$.

Proof. We transpose a classical argument (see [8]). Let $\left(\mu_{n}\right)$ be a decreasing series converging to zero. For a fixed $t>0$, consider $H_{-n}=\mu_{n} U\left(\mu_{n}, t\right)$ for $n \in \mathbb{N}$. Then $\left(H_{n}\right)_{n \leq 0}$ is a $\mathcal{H}$-reversed martingale, where $\mathcal{H}_{n}=\sigma\left(H_{m}, m \leq n\right)$; hence it converges a.s. and in $L^{1}$ (see e.g. [17]). Indeed, if $m<n$, any $\mu_{m}$-upcrossing contains a $\mu_{n}$-upcrossing, and the probability that a given $\mu_{n^{-}}$ upcrossing is contained in a $\mu_{m}$-upcrossing is $\mu_{n} / \mu_{m}$ (which is the probability that $R$, started from level $\mu_{n}$, reaches level $\mu_{m}$ before returning to zero). Setting $\left(\mu_{m}\right)=\left(2^{-m}\right)$, this defines a local time for $\left(X_{1}, X_{2}\right)$ at $(0,0)$; in particular $(0,0)$ is a regular point $(4<\kappa<8)$.

The stability index is a straightforward consequence of Brownian scaling for $\left(X_{1}, X_{2}\right)$ and the homogeneity property of $r$. More precisely, let $\lambda>0$; define $\tilde{X}_{i, t}=\lambda^{-1} X_{i, \lambda^{2} t}$, so that $\left(\tilde{X}_{1}, \tilde{X}_{2}\right)$ and $\left(X_{1}, X_{2}\right)$ have the same law. Then, if $\tilde{U}$ designates the number of upcrossings for $\tilde{R}=r\left(\tilde{X}_{1}, \tilde{X}_{2}\right)$, one has

$$
\tilde{U}(h, t)=U\left(h \lambda^{4-\kappa / 2}, \lambda^{2} t\right) .
$$


Let $\mu$ be the decreasing sequence such that $\left\{\mu_{m}\right\}_{m \geq 0}=\left\{2^{-m}\right\}_{m \geq 0} \cup\left\{\lambda^{\kappa / 2-4} 2^{-m}\right\}_{m \geq 0}$. Applying the above result, one gets the a.s. identity: $\tilde{\ell}_{t}=\lambda^{\kappa / 2-4} \ell_{\lambda^{2} t}$. Since $\ell, \tilde{\ell}$ are identical in law, this implies that right-inverse of $\ell$, which is a subordinator by construction, is a stable subordinator with index $(2-\kappa / 4)$.

By construction, the set of cut-times of the original $\operatorname{SLE}(\kappa, \kappa-4, \kappa-4)$ (i.e. times at which the trace lies both on the left and right boundaries of the final hull $\left.K_{\infty}\right)$ is the zero set of the process $\left(X_{1}, X_{2}\right)$.

Corollary 13. The set of cut-times of $\operatorname{SLE}(\kappa, \kappa-4, \kappa-4)$ has a.s. Hausdorff dimension $(2-\kappa / 4), 4<\kappa<8$. More precisely, the $\tilde{\varphi}_{\kappa}$-measure of cut-times is a.s. positive and locally finite, where:

$$
\tilde{\varphi}_{\kappa}(\varepsilon)=\varepsilon^{2-\kappa / 4}(\log |\log \varepsilon|)^{\kappa / 4-1} .
$$

Moreover, the $\tilde{\varphi}_{\kappa}$-measure of cut-times up to time $t$ is a multiple of the local time of $\left(X_{1}, X_{2}\right)$ at $(0,0)$.

Proof. Since the right-continuous inverse of $\ell$ is a stable subordinator with index $(2-\kappa / 4)$, the zero set of $\left(X_{1}, X_{2}\right)$ has the same distribution as the zero set of a semi-stable process with index $(\kappa / 4-1)^{-1}$, and the result follows from [21].

Let $\tau$ be the right-continuous of $\ell$. As $\tau$ is stable, one can formulate excursion-type results w.r.t. cut-times. Note that the final hull of an $\operatorname{SLE}(6,2,2)$ is identical in law to the filling of a Brownian excursion (see [7, Section 6), since these are two realizations of the (unique) restriction measure with index 1 (see 11). So, for $\kappa=6$, the corollary we presently state connects with results in 22 .

Corollary 14. (i) The process $\left(H_{u}, w_{u}\right)_{u}=\left(K_{\tau_{u}}, W_{\tau_{u}}\right)_{u}$ is a $\mathcal{Q} \times \mathbb{R}$-valued stable Lévy process, with index $(2-\kappa / 4)$. Moreover, for $\kappa \geq 6$, the following restriction formula holds: if $A$ is a smooth + -hull, $\alpha=\alpha(\kappa, 2 \kappa-8)=(\kappa-$ $2)(\kappa-3) / 2 \kappa$, and $L$ is an independent loop-soup with intensity $\lambda_{\kappa}$, then :

$$
\phi_{A}^{\prime}(0)^{\alpha}=\mathbb{E}\left(\phi_{\phi_{H_{u}}(A)}^{\prime}\left(w_{u}\right)^{\alpha} \mathbf{1}_{H_{u} \cap A^{L}=\varnothing}\right) .
$$

(ii) Let $e_{u}=\left(g_{\tau_{u^{-}}}\left(K_{\tau_{u}} \backslash K_{\tau_{u^{-}}}\right), W_{\tau_{u}}-W_{\tau_{u^{-}}}\right)$if $\tau_{u}>\tau_{u^{-}}$, and $e_{u}=\partial$ if $\tau_{u}=\tau_{u^{-}}$. Then $\left(e_{u}\right)_{u}$ is a $(\mathcal{Q} \times \mathbb{R}) \cup\{\partial\}$-valued, $(\mathcal{X} \vee \mathcal{Y})_{\tau_{u}}$ - Poisson process.

Proof. (i) As in the one-sided case, this follows from the fact that if $T$ is a $\mathcal{X} \vee \mathcal{Y}$ stopping time, with $X_{1, T}=X_{2, T}=0$ a.s., then:

$$
\left(g_{T+s} \circ g_{T}^{-1}\left(W_{T}^{-}\right)-W_{T+s}, g_{T+s} \circ g_{T}^{-1}\left(W_{T}^{+}\right)-W_{T+s}, X_{1, T+s}, X_{2, T+s}\right)_{s}
$$

is distributed as $\left(Y_{1, t}, Y_{2, t}, X_{1, t}, X_{2, t}\right)_{t}$ started from $\left(0^{-}, 0^{+}, 0^{-}, 0^{+}\right)$and is independent from $(\mathcal{X} \vee \mathcal{Y})_{T}$ (see proof or Proposition 3). The restriction formula is then a consequence of [7], Section 6. Assertion (ii) follows immediately. 
As in the one-sided case, one can conjecture that the Lévy process $\left(K_{\tau_{u}}, W_{\tau_{u}}\right)_{u}$ is characterized (up to a scale factor) by the stability property and the restriction formula (if $\kappa \geq 6$ ). In the case $\kappa=6$, since $\lambda_{6}=0$, the law of the total hull is characterized by the restriction formula.

Finally, we list some remaining questions. It is very likely that $\mathcal{X}^{i} \vee \mathcal{Y}=$ $\mathcal{X}^{i}, i=1,2$, though proving this seems a bit messy. Getting a constructive (Schramm-Loewner) description of the excursion measure ("bead measure") does not look quite straightforward, and may help to understand the law of $\left(\tau_{u}, W_{\tau_{u}}\right)_{u}$, where the first marginal is a stable subordinator with index $(2-\kappa / 4)$ and the second marginal is a symmetric stable process with index $(4-\kappa / 2)$. A last problem, essentially equivalent to describing the bead measure, concerns beads "conditioned to have infinite lifetime"; the first step consists in taking the Doob $h$-transform of the process $\left(X_{1}, X_{2}\right)$, using the "scale function" $r$. In particular, for $\kappa=6$, it should give (for an appropriate conditioning procedure) a restriction measure with index 2 (see [25]).

\section{Proof of Watts' formula}

In this section, we present a proof of Watts' formula, that describes the probability that there exists a double crossing in a rectangle (top-bottom and left-right) in the scaling limit of critical percolation. This formula was derived by Watts using (non-rigorous) Conformal Field Theory techniques (23]). In [6], Section 5 , we discussed how Watts' formula could be rephrased in $\mathrm{SLE}_{6}$ terms. We now sum up this discussion, for the reader's convenience.

Consider a Jordan domain $(D, a, b, c, d)$ with four points marked on the boundary (in counterclockwise order, say). Suppose that a portion of the triangular lattice with mesh $\varepsilon$ approximates this domain; each site of the lattice is colored in blue or yellow with probability $1 / 2$, and all sites are independent (this is critical site percolation on the triangular lattice). Denote by $C_{b}(A, B)$ (resp. $C_{y}(A, B)$ ) the fact that two site subsets $A$ and $B$ are connected by a blue (resp. yellow) path, and $T_{b}(A, B, C)$ (resp. $T_{y}(A, B, C)$ ) the fact that $A, B$ and $C$ are all connected by a blue (resp. yellow) cluster of sites. Cardy's formula gives the probability of events of type $C_{b}((a b),(c d))$ in the scaling limit $(\varepsilon \searrow 0)$, and similarly Watts' formula gives the probability of the event $\left\{C_{b}((a b),(c d)), C_{b}((b c),(d a))\right\}$. For plane topology reasons, it appears that:

$$
\begin{gathered}
\left\{C_{b}((a b),(c d)), C_{b}((b c),(d a))\right\}=\left\{T_{b}((a b),(b c),(c d))\right\} \backslash\left\{C_{y}((a b),(c d))\right\} \\
\left\{T_{b}((a b),(b c),(c d))\right\}=\left\{C_{b}((a b),(c d))\right\} \backslash\left\{C_{b}((a b),(c d)), T_{y}((a b),(b c),(c d))\right\} .
\end{gathered}
$$

Since switching the colors of all the sites is a measure-preserving operation, it follows that:

$\mathbb{P}\left(C_{b}((a b),(c d)), C_{b}((b c),(d a))\right)=\mathbb{P}\left(C_{b}((a b),(c d))\right)-2 \mathbb{P}\left(T_{b}((a b),(b c),(c d)), C_{y}((a b),(c d))\right)$. 
Consider now a Jordan domain $(T, a, b, c)$ with three points marked on the boundary, and suppose that the sites on $(a b)$ are set to blue and the sites on $(c a)$ are set to yellow (the arc $(b c)$ remaining free). Then one can define an exploration process starting from $a$ and stopped when it reaches $(b c)$; this exploration process is the interface between blue sites connected to ( $a b)$ and yellow sites connected to $(c a)$. As the mesh goes to zero, Smirnov's results (20]) imply that this exploration process converges to chordal $\operatorname{SLE}(6)$ in $(D, a, x)$ stopped when it hits $(b c)$, where $x$ can be chosen arbitrarily on $(b c)$ (which reflects the locality property of $\operatorname{SLE}(6))$.

Let $X$ be the random endpoint of this exploration process (i.e. $X$ is the first point on $(b c)$ reached by this process). If $(T, a, b, c)$ is an equilateral triangle, then the distribution of $X$ is uniform on $(b c)$; this is Carleson's approach of Cardy's formula. Let $D$ (resp. E) be the lowest point on $(a b)$ (resp. on $(c a)$ ) reached by the process before $X$. The exploration hull defines a (random) conformal quadrilateral $(K, a, D, X, E)$. From the self-duality of the triangular lattice, this quadrilateral is either crossed by a yellow path connecting $(a D)$ and $(X E)$ (in which case the exploration process visits $E$ before $D$ ) or by a blue path connecting $(D X)$ and $(E a)$ (and $D$ is visited before $E$ ).

Now, let $x$ be some point on $(b c)$. Then, the event $C_{b}((a b),(x c)$ ) (with free boundary conditions) is equivalent to $X \in(x c)$. Moreover, the event $\left\{T_{y}((a b),(x c),(c a)), C_{b}((a b),(x c))\right\}$ is equivalent to $X \in(x c)$ and $E$ is visited before $D$. Hence:

$$
\begin{array}{rrr}
\mathbb{P}\left(C_{b}((a b),(x c)), C_{b}((b x),(c a))\right)= & \mathbb{P}\left(C_{b}((a b),(x c))\right)-2 \mathbb{P}\left(T_{y}((a b),(x c),(c a)), C_{b}((a b),(x c))\right) \\
= & \mathbb{P}(X \in(x c))-2 \mathbb{P}(X \in(x c), E \text { visited before } D) .
\end{array}
$$

Recall that chordal $\mathrm{SLE}_{6}$ is the scaling limit of percolation interfaces for critical site percolation on the triangular lattice (see [20]). More precisely, consider a chordal $\mathrm{SLE}_{6}$ in $(\mathbb{H}, 0, \infty)$; here $(T, a, b, c)=(\mathbb{H}, 0,1, \infty)$. Then the distribution of $\gamma_{\tau_{1}}=\inf (\gamma \cap(1, \infty))$ is given by Cardy's formula:

$\mathbb{P}\left(1<\gamma_{\tau_{1}}<x\right)=B(1 / 3,1 / 3)^{-1} \int_{1 / x}^{1} \frac{d s}{(s(1-s))^{2 / 3}}=B(1 / 3,1 / 3)^{-1} \int_{1}^{x} \frac{d s}{(s(s-1))^{2 / 3}}$

for any $x>1$. Now, let $g=\sup \left(t<\tau_{1}: \gamma_{t} \in \mathbb{R}\right)$ be the last time before $\tau_{1}$ spent by the trace on the real line. Then Watts' formula can be rephrased as follows:

$$
\mathbb{P}\left(\gamma_{g}<0 \mid \gamma_{\tau_{1}} \in d x\right)=B(2 / 3,2 / 3)^{-1} \int_{1 / x}^{1} \frac{d s}{(s(1-s))^{1 / 3}} d x .
$$

This conditional probability can be derived from the study of $\operatorname{SLE}(6,2,2)$, as we presently explain. Notations $(d, k, \Upsilon, \ldots)$ are as in Section 3.

Lemma 15. Consider an $\operatorname{SLE}(6,2,2)$ process in $(\mathbb{H}, 0, \infty)$, started from $\left(0, y_{1}, y_{2}\right)$, where $y_{1}<0<y_{2}$. Let $X_{1}$ be the leftmost swallowed point on $\left(y_{1}, 0\right)$ and $X_{2}$ the 
rightmost swallowed point on $\left(0, y_{2}\right)$. Then the probability that the SLE reaches $X_{1}$ before $X_{2}$ is given by

$$
B(2 / 3,2 / 3)^{-1} \int_{0}^{t} \frac{d s}{(s(1-s))^{1 / 3}}
$$

where $t=y_{2} /\left(y_{2}-y_{1}\right)$.

Proof. The first part of the proof holds for a general value of $\kappa>4$. We have seen that the distribution of $\left(X_{1}, X_{2}\right)$ is $\Upsilon\left(\left(y_{1}, y_{2}\right),.\right)$. Let $\tau=\tau_{1} \wedge \tau_{2}$, where $\tau_{i}$ is the first time the trace reaches $X_{i}, i=1,2$. Conditionally on $\left(X_{1}, X_{2}\right)$, the process $\left(g_{t}\left(X_{1}\right)-W_{t}, g_{t}\left(X_{2}\right)-W_{t}\right)$, stopped at time $\tau$, is Markov with semigroup $Q^{\downarrow \downarrow}$. From scale invariance, one can write

$$
\mathbb{P}_{\left(x_{1}, x_{2}\right)}^{\downarrow \downarrow}\left(\tau_{1}<\tau_{2}\right)=f\left(x_{2} /\left(x_{2}-x_{1}\right)\right)
$$

for some function $f$ with $f(0)=0, f(1)=1$. It is then standard that $\left(f\left(X_{2, t} /\left(X_{2, t}-\right.\right.\right.$ $\left.\left.X_{1, t}\right)\right)_{t \geq 0}$ is a martingale (in the $\mathcal{X} \vee \mathcal{Y}$ filtration). From the definition of $Q^{\downarrow \downarrow}$, it appears that $f$ is such that

$$
g_{t}^{\prime}\left(X_{1}\right) g_{t}^{\prime}\left(X_{2}\right) f\left(X_{2, t} /\left(X_{2, t}-X_{1, t}\right)\right) \partial_{12} k\left(X_{1, t}, X_{2, t}\right)
$$

is a $\mathbb{P}$-local martingale, conditionally on $\left(X_{1}, X_{2}\right)$. So $\left(x_{1}, x_{2}\right) \mapsto f\left(x_{2} /\left(x_{2}-x_{1}\right)\right)$ annihilates the differential operator:

$$
\begin{aligned}
& \left(\partial_{12} k\right)^{-1}\left(\frac{\kappa}{2}\left(\partial_{1}+\partial_{2}\right)^{2}+\frac{2}{x_{1}} \partial_{1}+\frac{2}{x_{2}} \partial_{2}-\frac{2}{x_{1}^{2}}-\frac{2}{x_{2}^{2}}\right)\left(\partial_{12} k\right)= \\
& \frac{\kappa}{2}\left(\partial_{1}+\partial_{2}\right)^{2}+\frac{2}{x_{1}} \partial_{1}+\frac{2}{x_{2}} \partial_{2}-\left(\frac{1}{x_{1}}+\frac{1}{x_{2}}\right) \frac{4+(4-\kappa)(4-\kappa / 2) x_{1} x_{2} /\left(x_{2}-x_{1}\right)^{2}}{1+(4-\kappa / 2) x_{1} x_{2} /\left(x_{2}-x_{1}\right)^{2}}\left(\partial_{1}+\partial_{2}\right) .
\end{aligned}
$$

It follows that $f$ solves the ODE:

$$
f^{\prime \prime}(t)+\left(-\frac{4}{\kappa}\left(\frac{1}{t}+\frac{1}{t-1}\right)+2 \frac{(4-\kappa / 2)(2 t-1)}{1+(4-\kappa / 2) t(t-1)}\right) f^{\prime}(t)=0
$$

which implies that

$$
f(t)=c \int_{0}^{t} \frac{(s(1-s))^{4 / \kappa}}{(1+(4-\kappa / 2) s(s-1))^{2}} d s
$$

where $c$ is such that $f(1)=1$. Let

$$
h\left(y_{1}, y_{2}\right)=\mathbb{P}_{\left(y_{1}, y_{2}\right)}^{\uparrow \uparrow}\left(\tau_{1}<\tau_{2}\right) .
$$

Since the distribution of $\left(X_{1}, X_{2}\right)$ is $\Upsilon\left(\left(y_{1}, y_{2}\right),.\right)$, and the conditional probability of $\left\{\tau_{1}<\tau_{2}\right\}$ given $\left(X_{1}, X_{2}\right)$ is $f\left(X_{2} /\left(X_{2}-X_{1}\right)\right)$, one gets, integrating by 
parts:

$$
\begin{aligned}
h\left(y_{1}, y_{2}\right)= & \int_{y_{1}}^{0} \int_{0}^{y_{2}} f\left(\frac{x_{2}}{x_{2}-x_{1}}\right) \frac{-\partial_{12} k\left(x_{1}, x_{2}\right)}{k\left(y_{1}, y_{2}\right)} d x_{1} d x_{2} \\
= & \int_{y_{1}}^{0}\left(\frac{-\partial_{1} k\left(x_{1}, y_{2}\right)}{k\left(y_{1}, y_{2}\right)} f\left(\frac{y_{2}}{y_{2}-x_{1}}\right)-\int_{0}^{y_{2}} \frac{-\partial_{1} k\left(x_{1}, x_{2}\right)}{k\left(y_{1}, y_{2}\right)} \frac{-x_{1}}{\left(x_{2}-x_{1}\right)^{2}} f^{\prime}\left(\frac{x_{2}}{x_{2}-x_{1}}\right) d x_{2}\right) d x_{1} \\
= & f\left(\frac{y_{2}}{y_{2}-y_{1}}\right)+\int_{y_{1}}^{0} \frac{k\left(x_{1}, y_{2}\right)}{k\left(y_{1}, y_{2}\right)} f^{\prime}\left(\frac{y_{2}}{y_{2}-x_{1}}\right) \frac{y_{2}}{\left(y_{2}-x_{1}\right)^{2}} d x_{1} \\
& -\int_{y_{1}}^{0} \int_{0}^{y_{2}} \frac{\partial_{1} k\left(x_{1}, x_{2}\right)}{k\left(y_{1}, y_{2}\right)} \frac{x_{1}}{\left(x_{2}-x_{1}\right)^{2}} f^{\prime}\left(\frac{x_{2}}{x_{2}-x_{1}}\right) d x_{1} d x_{2} \\
= & f\left(\frac{y_{2}}{y_{2}-y_{1}}\right)+c \int_{y_{1}}^{0} \frac{\left(-x_{1}\right) y_{2}^{1+4 / \kappa}\left(y_{2}-x_{1}\right)^{\kappa / 2-6}}{y_{1}^{d}\left(y_{2}-y_{1}\right)^{\kappa d^{2} / 2}\left(1+(4-\kappa / 2)\left(x_{1} y_{2}\right) /\left(y_{2}-x_{1}\right)^{2}\right)^{2}} d x_{1} \\
& +c d \int_{y_{1}}^{0} \int_{0}^{y_{2}}\left(\frac{1}{x_{1}}+\frac{\kappa}{2} \frac{d}{x_{1}-x_{2}}\right) x_{1}^{2} x_{2} \frac{\left(x_{2}-x_{1}\right)^{\kappa / 2-6}}{\left(1+(4-\kappa / 2) x_{1} x_{2} /\left(x_{2}-x_{1}\right)^{2}\right)^{2}} \frac{d x_{1} d x_{2}}{k\left(y_{1}, y_{2}\right)} .
\end{aligned}
$$

Now, let $\kappa=6$. The two integrals in the former expression have rational integrands, so they can be computed mechanically. After simplifications, one gets:

$$
h\left(y_{1}, y_{2}\right)=f\left(\frac{y_{2}}{y_{2}-y_{1}}\right)+\frac{c}{3} \frac{y_{1} y_{2}\left(y_{1}+y_{2}\right)}{\left(y_{1}^{2}-y_{1} y_{2}+y_{2}^{2}\right)\left(-y_{1} y_{2}\right)^{1 / 3}\left(y_{2}-y_{1}\right)^{1 / 3}} .
$$

By homogeneity, $h\left(y_{1}, y_{2}\right)=\tilde{h}(t)$, where $t=y_{2} /\left(y_{2}-y_{1}\right)$. Differentiating the above expression:

$$
\begin{aligned}
c^{-1} \tilde{h}^{\prime}(t) & =\frac{(t(1-t))^{2 / 3}}{(1+t(t-1))^{2}}+\frac{d}{d t}\left(\frac{(1-2 t)(t(1-t))^{2 / 3}}{3(1+t(t-1))}\right) \\
& =\frac{2}{9}(t(1-t))^{-1 / 3}
\end{aligned}
$$

Hence $c^{-1}=2 B(2 / 3,2 / 3) / 9$, and

$$
\mathbb{P}_{\left(y_{1}, y_{2}\right)}^{\uparrow \uparrow}\left(\tau_{1}<\tau_{2}\right)=B(2 / 3,2 / 3)^{-1} \int_{0}^{t} \frac{d s}{(s(1-s))^{1 / 3}}
$$

where $t=y_{2} /\left(y_{2}-y_{1}\right)$.

Consider now critical site percolation on the triangular lattice. In this case, interfaces converge to $\mathrm{SLE}_{6}$ in the scaling limit (see [20]).

Proposition 16 (Watts' formula). The probability that the four boundary arcs of a conformal quadrilateral $(D, a, b, c, d)$, where $D$ is a simply connected Jordan domain, are connected by a cluster is:

$$
B(1 / 3,1 / 3)^{-1} \int_{0}^{z} \frac{1}{(s(1-s))^{2 / 3}}\left(1-2 B(2 / 3,2 / 3)^{-1} \int_{0}^{s} \frac{d r}{(r(1-r))^{1 / 3}}\right) d s
$$

where $z$ is the cross-ratio $[a, b, c, d]$. 
Proof. Consider a chordal SLE(6) in $(\mathbb{H}, 0, \infty) ; \gamma$ is the trace, $\tau_{1}$ is the swallowing time of 1 , and $g$ is the last time before $\tau_{1}$ spent by the trace on $\mathbb{R}$. As we have seen, $\left(1 / \gamma_{\tau_{1}}\right)$ has distribution $\operatorname{Beta}(1 / 3,1 / 3)$ (Cardy's formula), and Watts' formula is equivalent to

$$
\mathbb{P}\left(\gamma_{g}<0 \mid \gamma_{\tau_{1}} \in d x\right)=B(2 / 3,2 / 3)^{-1} \int_{1 / x}^{1} \frac{d s}{(s(1-s))^{1 / 3}} d x .
$$

So let $\left(x_{1}, x_{2}\right)$ be a neighbourhood of $x>1$. From the locality property of $\operatorname{SLE}(6)$ (see e.g. [24]), the $\operatorname{SLE}(6)$ conditionally on $\gamma_{\tau_{1}} \in\left(x_{1}, x_{2}\right)$ stopped at $\tau_{x_{1}}$ is identical in law to a time-changed chordal $\operatorname{SLE}(6)$ in $(\mathbb{H}, 0, x)$ with the corresponding conditioning, stopped when its trace hits $\left(x_{1}, x_{2}\right)$. Consider the homography $\phi(z)=z(1-x) /(z-x)$. Then the image of this conditioned SLE under $\phi$ is a chordal $\operatorname{SLE}(6)$ in $(\mathbb{H}, 0, \infty)$, conditioned on its trace not hitting $\left(y_{2}, 1-x\right) \cup\left(1, y_{1}\right)$, where $y_{i}=\phi\left(x_{i}\right), i=1,2$. What we have to compute is the probability that the trace is on $(1-x, 0)$ the last time it visits $(1-x, 1)$.

As $x_{1} \nearrow x, x_{2} \searrow x$, it appears that $y_{1} \rightarrow \infty, y_{2} \rightarrow-\infty$; formally, we have now an SLE(6) conditioned on its trace not hitting $(-\infty, 1-x) \cup(1, \infty)$. As we have seen, this singular conditioning can be realized as an $\operatorname{SLE}(6,2,2)$ process started from $(0,1-x, 1)$. According to the previous lemma, the probability that the trace is on $(1-x, 0)$ the last time it visits $(1-x, 1)$, for an $\operatorname{SLE}(6,2,2)$ process, is:

$$
B(2 / 3,2 / 3)^{-1} \int_{1 / x}^{1} \frac{d s}{(s(1-s))^{1 / 3}}
$$

which is exactly what we need. To make the previous limiting argument more precise, one can argue along the lines of Theorem 3.1 in [13], as we now sketch. If $\left(g_{t}\right)$ denotes the family of conformal equivalences associated with a chordal $\operatorname{SLE}(\kappa)$ process, $\kappa>4$, and $W$ is its driving process, let

$$
Z_{t}=\frac{W_{t}-g_{t}\left(u_{1}\right)}{g_{t}\left(u_{2}\right)-g_{t}\left(u_{1}\right)}
$$

for some $u_{1}<0<u_{2}$. Then, after an appropriate time-change, $Z$ is a diffusion on $[0,1]$ with leading eigenvector $h(z)=(z(1-z))^{1-4 / \kappa}$ (see [13]). Using this eigenvector, one can "condition" this diffusion to have infinite lifetime (i.e. $Z$ never swallows 0 or 1 , that is the SLE never swallows $u_{1}$ or $u_{2}$ ). Working backwards, it appears that the corresponding conditional SLE is precisely $\operatorname{SLE}(\kappa, \kappa-4, \kappa-4)$ started from $\left(0, u_{1}, u_{2}\right)$. Suppose now that $M \gg 1$; if a chordal $\operatorname{SLE}(\kappa)$ run until time $M^{2}$ has not swallowed $u_{1}, u_{2}$ yet, then the conditional probability that the trace does not hit $\left(-M, u_{1}\right) \cup\left(u_{2}, M\right)$ is bounded away from 0 . Conversely, if the trace does not hit $\left(-M, u_{1}\right) \cup\left(u_{2}, M\right), M \rightarrow \infty$, then the lifetime of $Z$ goes to infinity. So the two procedures - conditioning on the trace not hitting $\left(-M, u_{1}\right) \cup\left(u_{2}, M\right), M \rightarrow \infty$, or conditioning on $Z$ not hitting 0 or 1 before time $T, T \rightarrow \infty$ - yield the same limiting object, namely $\operatorname{SLE}(\kappa, \kappa-4, \kappa-4)$.

Alternatively, one can build on the two following facts (for $4<\kappa<8$ ): 
- Let $0<x<y, \tau_{x}$ the swallowing time of $x$. A chordal SLE $(\kappa)$ process in $(\mathbb{H}, 0, \infty)$ conditioned on $\gamma_{\tau_{x}} \in d y$ and stopped at $\tau_{y}$ is an $\operatorname{SLE}(\kappa, \kappa-4,-4)$ process in $(\mathbb{H}, 0, \infty)$ started from $(0, x, y)$ and stopped at $\tau_{y}$.

- A chordal $\operatorname{SLE}\left(\kappa, \rho_{1}, \rho_{2}\right)$ in $(\mathbb{H}, 0, \infty)$ started from $(0, x, y)$ is a timechanged $\operatorname{SLE}\left(\kappa, \kappa-6-\rho_{1}-\rho_{2}, \rho_{1}\right)$ in $(\mathbb{H}, 0, y)$ started from $(0, \infty, x)$.

The first fact is a direct consequence of Cardy's formula for SLE and Girsanov's theorem. The second fact can be proved by a computation along the lines of [11, Section 5. Consequently, an $\operatorname{SLE}(\kappa)$ conditioned on $\gamma_{\tau_{x}} \in d y$ is a time-changed $\operatorname{SLE}(\kappa, 2, \kappa-4)$ in $(\mathbb{H}, 0, y)$ started from $(0, \infty, y)$. For $\kappa=6$, one recovers the required result.

Originally, Watts identified this function as the only function annihilating the fifth-order differential operator

$$
(z(1-z))^{-2} \frac{d^{3}}{d z^{3}}(z(1-z))^{4 / 3} \frac{d}{d z}(z(1-z))^{2 / 3} \frac{d}{d z}
$$

and satisfying appropriate boundary conditions. This operator seems to have no obvious interpretation in the $\mathrm{SLE}_{6}$ framework. As pointed out in [9], the solution space of the associated ODE is spanned by: the constant function 1, the function appearing in Cardy's formula, the function appearing in Watts' formula, $z \mapsto \log (z), z \mapsto \log (1-z)$. Moreover, Cardy's prediction for the expected number of disjoint clusters connecting two opposite sides of a rectangle also belongs to this solution space (4], see also [14]).

It is also possible to express Watts' formula using equianharmonic elliptic functions. From the known value of the equianharmonic $\sigma$ function at halfperiods, one can deduce that Watts' formula imply the following result (14]).

Corollary 17. The probability that there exists a double crossing in a square is

$$
\frac{1}{4}+\frac{\sqrt{3}}{4 \pi}(3 \log 3-4 \log 2) \simeq 0.322120455 \ldots
$$

Note that the numerical value of this probability agrees with the estimate in [10].

\section{References}

[1] V. Beffara. Hausdorff dimensions for $\mathrm{SLE}_{6}$. preprint, arXiv:math.PR/0204208, 2002.

[2] J. Bertoin. Complements on the Hilbert transform and the fractional derivative of Brownian local times. J. Math. Kyoto Univ., 30(4):651-670, 1990. 
[3] J. Bertoin. Excursions of a $\mathrm{BES}_{0}(d)$ and its drift term $(0<d<1)$. Probab. Theory Related Fields, 84(2):231-250, 1990.

[4] J. L. Cardy. Conformal invariance and percolation. preprint, arXiv:mathph/0103018, 2001.

[5] P. Carmona, F. Petit, and M. Yor. Beta-gamma random variables and intertwining relations between certain Markov processes. Rev. Mat. Iberoamericana, 14(2):311-367, 1998.

[6] J. Dubédat. Reflected brownian motions, intertwining relations and crossing probabilities. Ann. Inst. H. Poincaré Probab. Statist., to appear, 2003.

[7] J. Dubédat. $\operatorname{SLE}(\kappa, \rho)$ martingales and duality. Ann. Probab., to appear, 2003.

[8] K. Itô and H. P. McKean, Jr. Diffusion processes and their sample paths. Springer-Verlag, Berlin, 1974. Second printing, corrected, Die Grundlehren der mathematischen Wissenschaften, Band 125.

[9] P. Kleban and D. Zagier. Crossing probabilities and modular forms. $J$. Statist. Phys., 113(3-4):431-454, 2003.

[10] R. Langlands, P. Pouliot, and Y. Saint-Aubin. Conformal invariance in two-dimensional percolation. Bull. Amer. Math. Soc. (N.S.), 30(1):1-61, 1994.

[11] G. Lawler, O. Schramm, and W. Werner. Conformal restriction: the chordal case. J. Amer. Math. Soc., 16(4):917-955 (electronic), 2003.

[12] G. F. Lawler, O. Schramm, and W. Werner. Conformal invariance of planar loop-erased random walks and uniform spanning trees. Ann. Inst. H. Poincaré Statist. Probab., to appear., 2002.

[13] G. F. Lawler, O. Schramm, and W. Werner. Values of Brownian intersection exponents. III. Two-sided exponents. Ann. Inst. H. Poincaré Probab. Statist., 38(1):109-123, 2002.

[14] R. S. Maier. On crossing event formulas in critical two-dimensional percolation. J. Statist. Phys., 111(5-6):1027-1048, 2003.

[15] J. Pitman and M. Yor. A decomposition of Bessel bridges. Z. Wahrsch. Verw. Gebiete, 59(4):425-457, 1982.

[16] D. Revuz and M. Yor. Continuous martingales and Brownian motion, volume 293 of Grundlehren der Mathematischen Wissenschaften. SpringerVerlag, Berlin, third edition, 1999.

[17] L. C. G. Rogers and D. Williams. Diffusions, Markov processes, and martingales. Vol. 1. Wiley Series in Probability and Mathematical Statistics: Probability and Mathematical Statistics. John Wiley \& Sons Ltd., Chichester, second edition, 1994. Foundations. 
[18] S. Rohde and O. Schramm. Basic Properties of SLE. Ann. Math., to appear, 2001.

[19] O. Schramm. Scaling limits of loop-erased random walks and uniform spanning trees. Israel J. Math., 118:221-288, 2000.

[20] S. Smirnov. Critical percolation in the plane. I. Conformal Invariance and Cardy's formula II. Continuum scaling limit. in preparation, 2001.

[21] S. J. Taylor and J. G. Wendel. The exact Hausdorff measure of the zero set of a stable process. Z. Wahrscheinlichkeitstheorie und Verw. Gebiete, 6:170-180, 1966 .

[22] B. Virág. Brownian beads. Probab. Theory Related Fields, 127(3):367-387, 2003.

[23] G. M. T. Watts. A crossing probability for critical percolation in two dimensions. J. Phys. A, 29(14):L363-L368, 1996.

[24] W. Werner. Lectures on random planar curves and Schramm-Loewner evolution. In Lecture Notes of the 2002 St-Flour summer school, to appear. Springer-Verlag, 2002.

[25] W. Werner. Girsanov transformation for $\operatorname{SLE}(\kappa, \rho)$ processes, intersection exponents and hiding exponent. Ann. Fac. Sci. Toulouse, 2003.

Laboratoire de Mathématiques, Bât. 425

Université Paris-Sud, F-91405 Orsay cedex, France

julien.dubedat@math.u-psud.fr 